\title{
بيان : نظام الاستعلامات في قواعد البيانات باللغة العربية
}
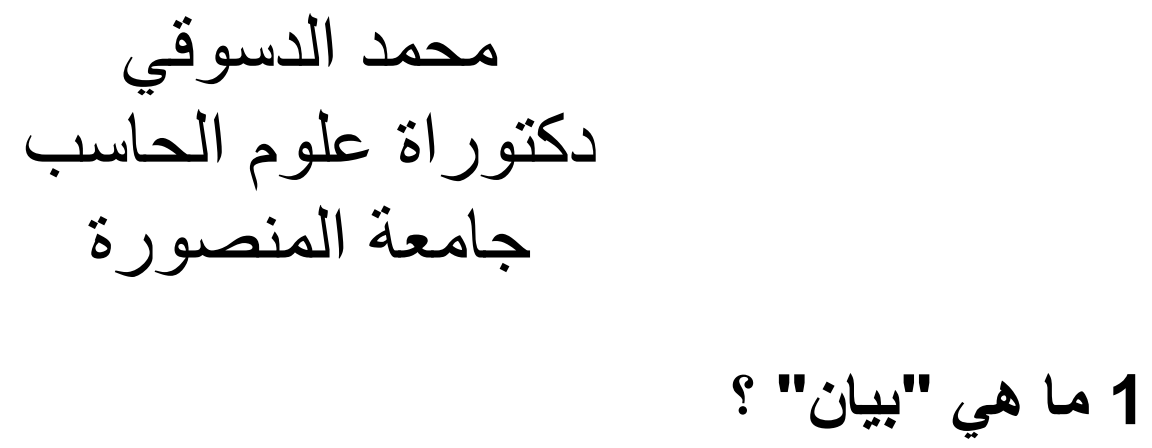

بيان هي نظام باللغة الطبيعية (العربية) للتنقيب المعلوماتي (Data Mining)، و هي تشبه وحدة التحليل و الاستعلام باللغة الإنجليزية (Analysis Component \& English Querying) في نظام Microsoft SQL Server

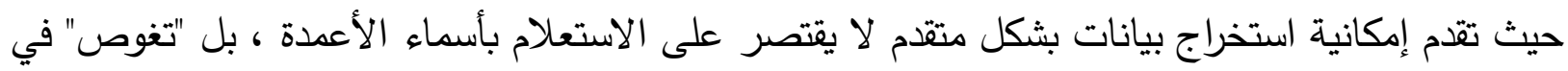
الجدول وتحلله ، لتسمح بالاستعلام بأحد قيم الخلايا.

\section{كما أنها تمتاز على هذه النظم بالآتي :}

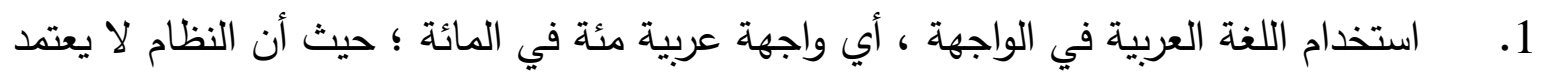

مطلقا على وحدة الإخراج ( Console ) ، و المستخدمة في إدخال جمل الاستعلام .

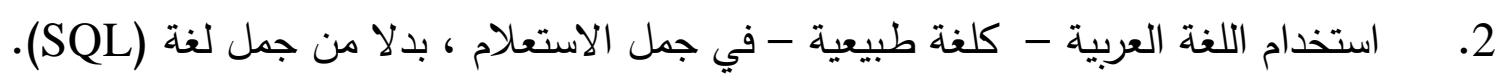

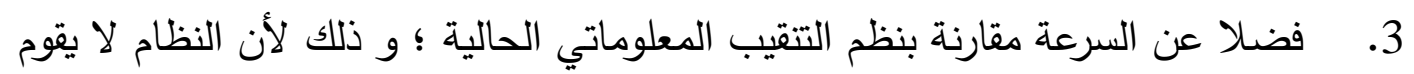

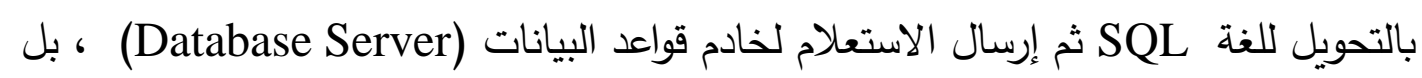

يقوم بالتنفيذ بنفسه . باليل

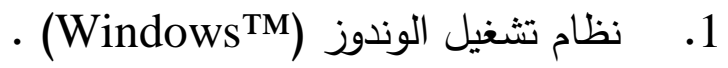

Visual Studio.Net .2

3. ذاكرة لا تقل عن 512 كيلوبايت . 


\section{3}

\section{1-3 فلسفة الواجهة}

تعتمد هذه الواجهة على الفلسفة العامة لبرامج الاستعلام من قواعد البيانات ، وذلك بتخصيص جزء

لعرض البيانات التي يتم التعامل معها، و آخر لإدخال جمل الاستعلام ، على أن يتم التحاور بين المستخدم و لإنها

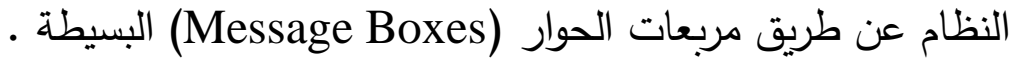

\section{2-3 لقطات من الواجهة (Snapshots)}

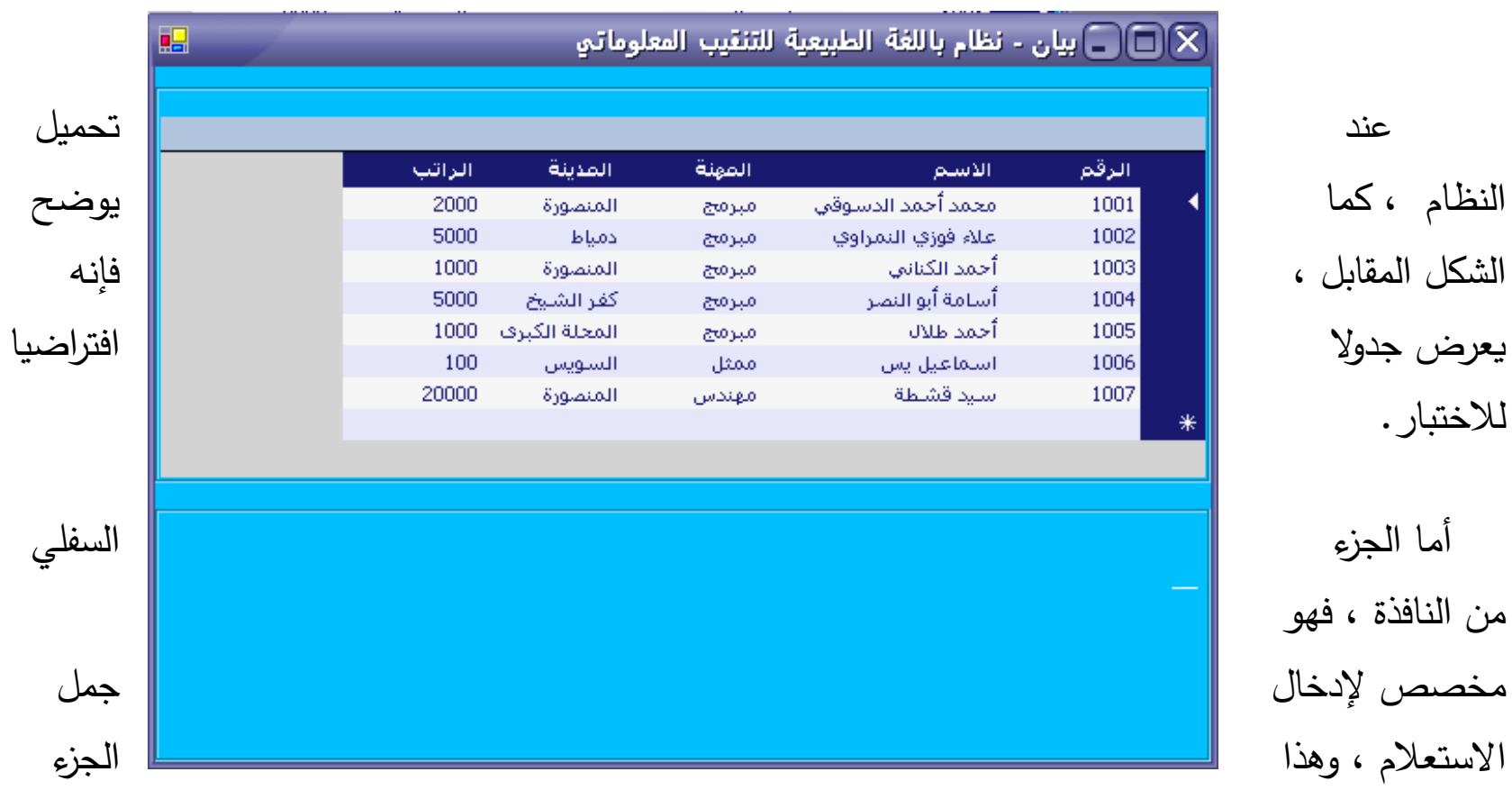

يتثابه مع تقنية "سطر الأوامر" الثهيرة (Command Line) .

و يمكن الرجوع إلى الجزء الخاص بنظام "مرن" ، للاستزادة من المعلومات الفنية حول تقنية سطر

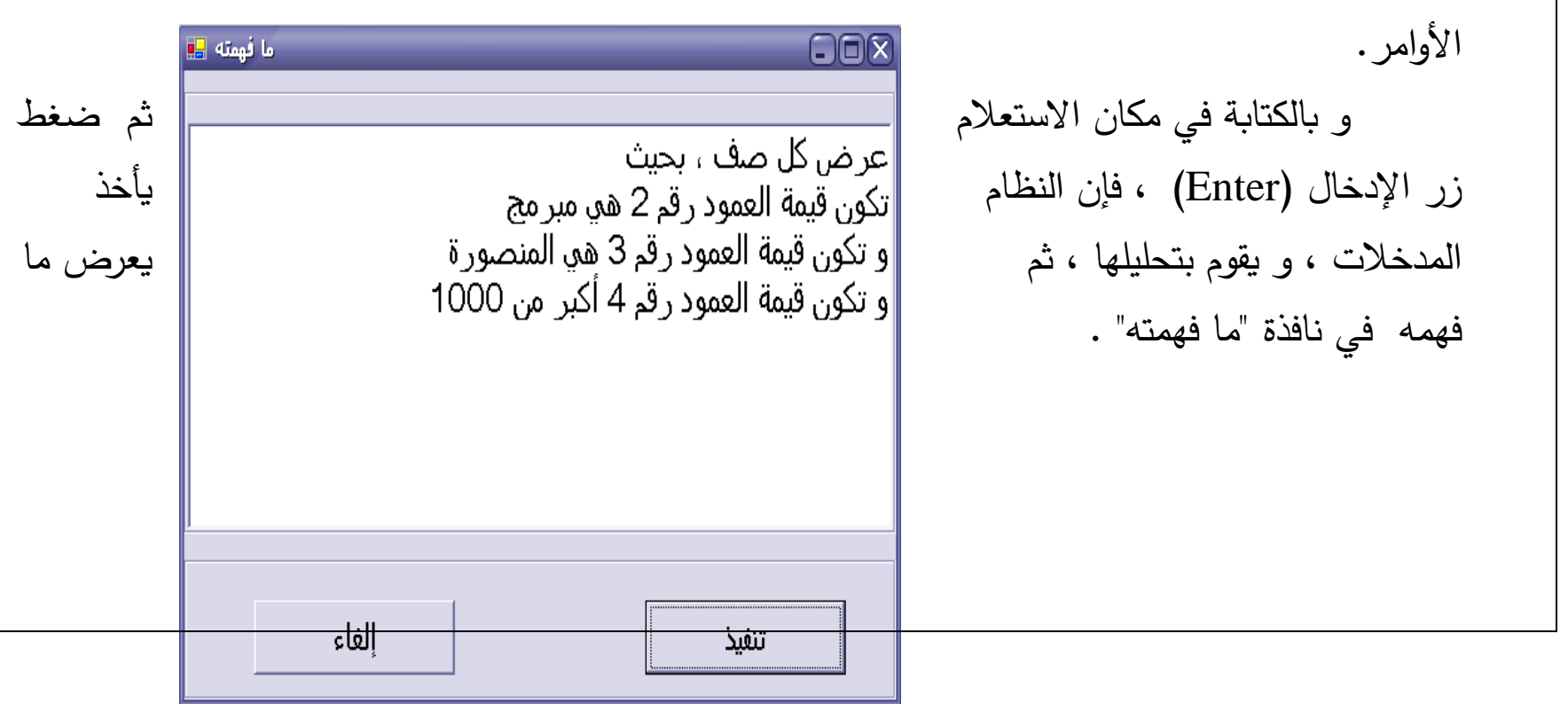




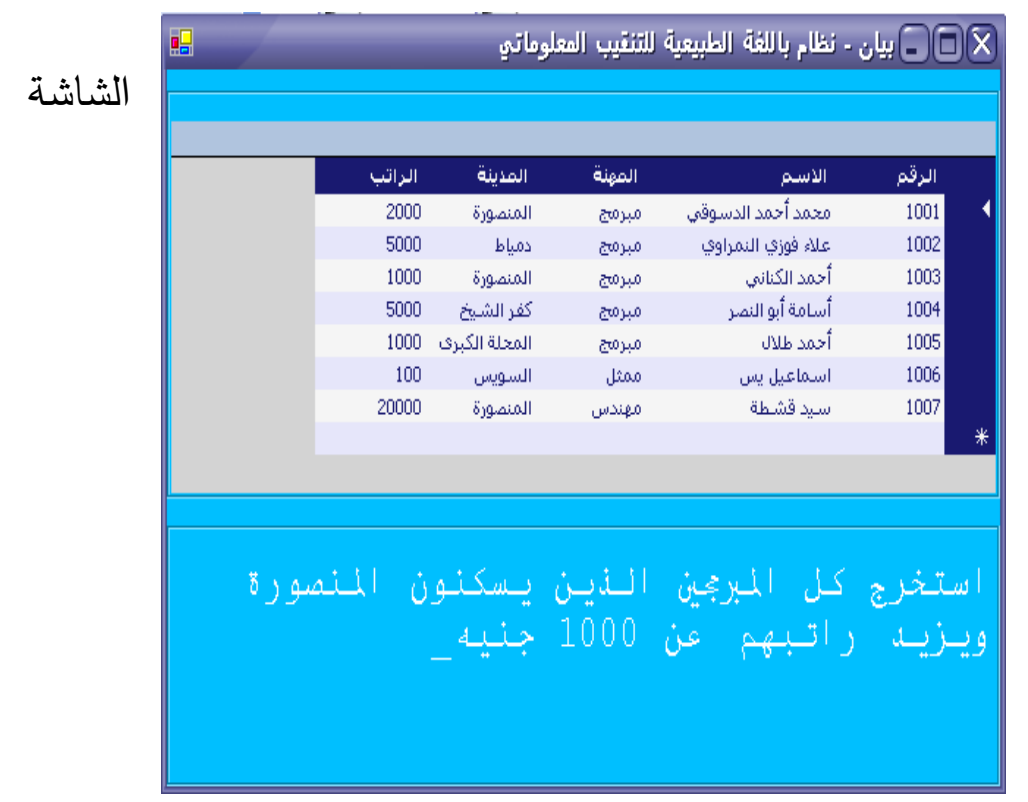

أسباب فصل الناتج عن الرئيسية :

o الوضوح و التبسيط

؛ فلا تزدحم

الشاشة ـ

O عدم ضياع أجزاء

من الناتج متعدد

السطور بسبب

خاصية الإزاحة

- (Scroll)

م توفير إمكانية التعامل مع ناتج الفهم بشكل مستقل عن الأوامر، مثل تخزين الناتج في ملف (انظر تخزين ناتج الفهم ) وتغيير خط الكتابة (انظر تهيئة ناتج الفهح ) •

وفي حالة "رضاء" المستخدم عن الناتج ، بمعنى أن النظام فهم ما طلبه المستخدم بالضبط، فيمكنه

أن يضغط زر "تنفيذ" ، ليحصل على ناتج جملة الاستعلام ، الذي يتم عرضه في نافذة مستقلة .

\begin{tabular}{||r|r|r|r|r|r||}
\hline & \\
\hline & & & & & \\
\hline
\end{tabular}

وكما نرى لا يوجد إلا صف واحد يحقق هذه الشروط مجتمعة ، وبالتالي يتم عرضه .

هذا إذا كانت جملة الاستعلام تقوم باستخراج بيانات ، أما إذا كانت تقوم بالتعديل في البيانات الأصلية كمثل : 
و التي يفهمها النظام على أنها :

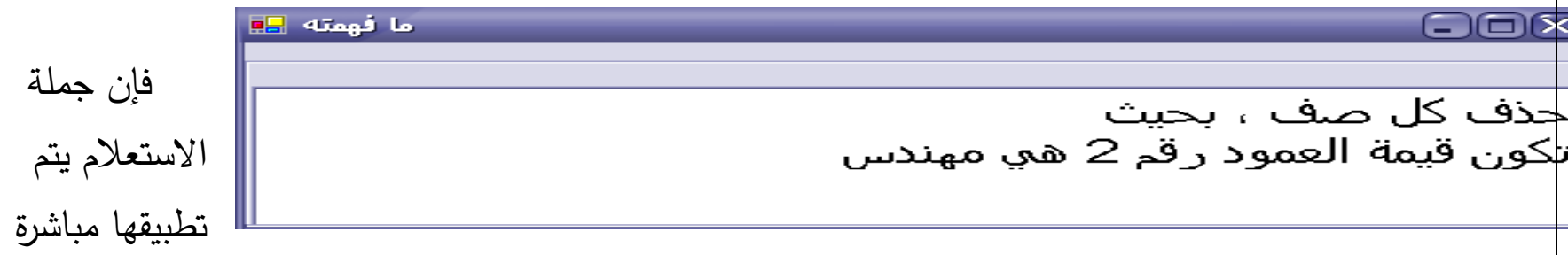

على الجدول الأصلي ، مسببة حذف كل صف تكون قيمة العمود رقم (2) فيه هي "مهند" ، أو بمعنى آخر "حذف كل المهندسين" .

يمكن النظر إلى المدخلات على أنها : عملية + شروط .

العملية قد تكون (عرض - حذف - تعديل ، ... ) .

الشروط قد تكون (قيمة خلية ، أو اسم عمود ، أو علاقات على قيمة خلية) .

ويقصد بالعلاقات على قيمة خلية ، جمل مثل :

أكبر من ، أصغر من ، أصغر من أو يساوي ،لا تساوي، تساوي، يزيد عن ، تقل عن ، ... الخ .

مع تعدد صيخ كتابة نفس الأمر ، فعلى سبيل المثال ، أمر الاستخراج يمكن كتابته بأفعال مختلفة

(استخرج ، عرض ، أريد، أعطني ، ...) ، بل و بصيخ مختلفة (أمر ، مصدر صريح ،مصدر مؤول،

فعل مضارع).

وبعمل تباديل من وجود/غياب عنصري المدخلات ، نجد أن حالات التشغيل تكون كما يلي :

\begin{tabular}{|c|c|c|}
\hline التصرف & 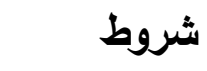 & 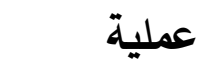 \\
\hline الحالة العادية . & موجودة & موجودة \\
\hline بلا عملية ، لا تتفيذ . & 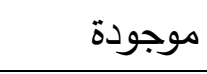 & 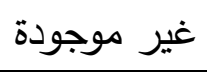 \\
\hline افتراض العملية على كل الصفوف . & 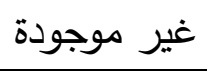 & 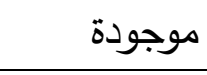 \\
\hline بلا مدخلات تقريبا . & غير موجودة & غير موجودة \\
\hline
\end{tabular}

وفيما يلي بيان للثلاث حالات الأخيرة .

3-3 حالات التشغيل الغير تقليدية 


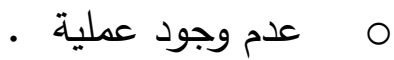

م وجود شروط .

كما في :

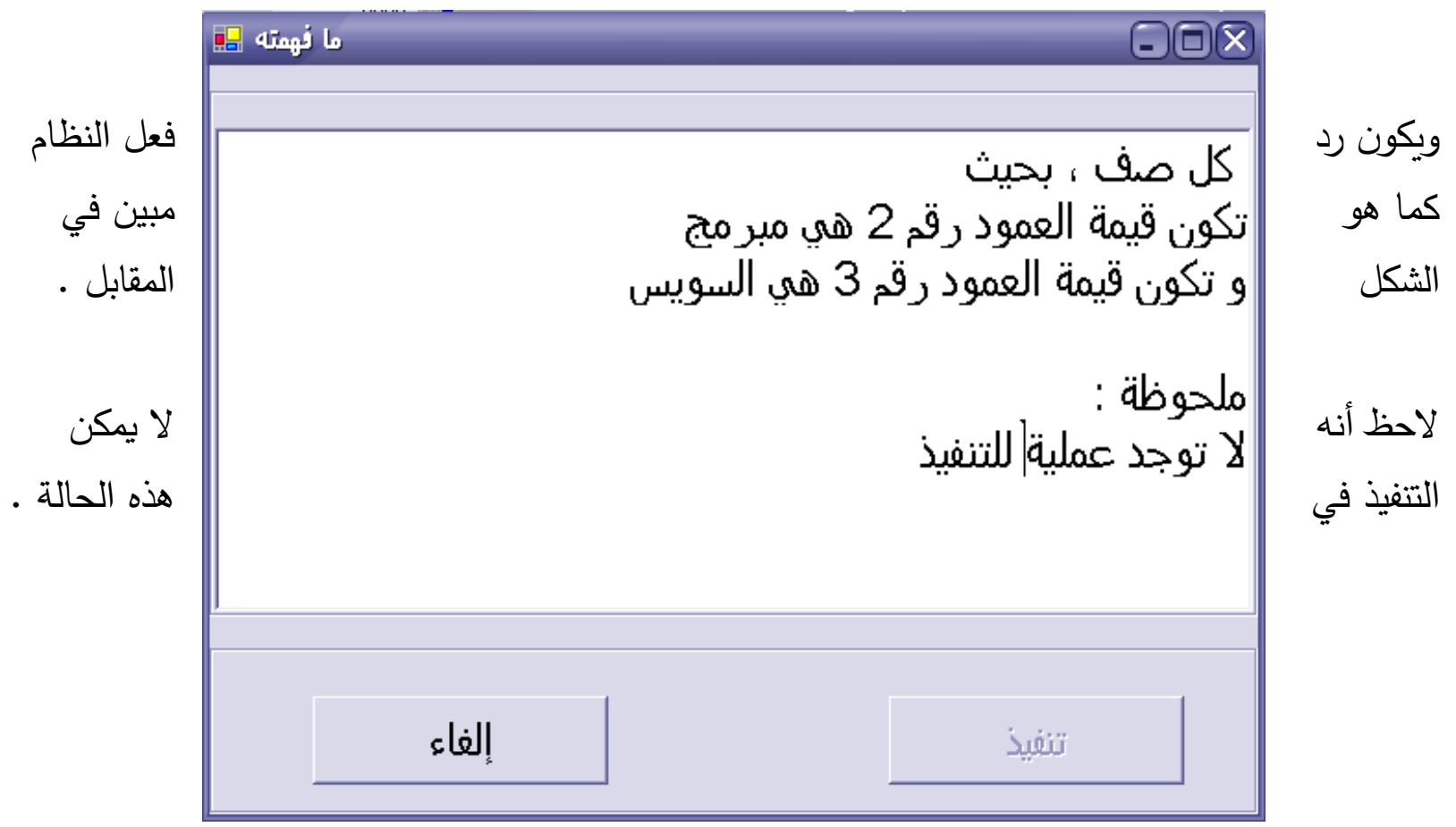

الحالة الثانية :

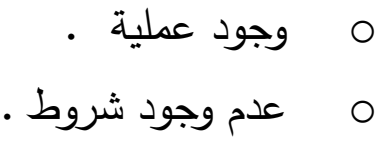

كما في : 
رد فعل النظام :

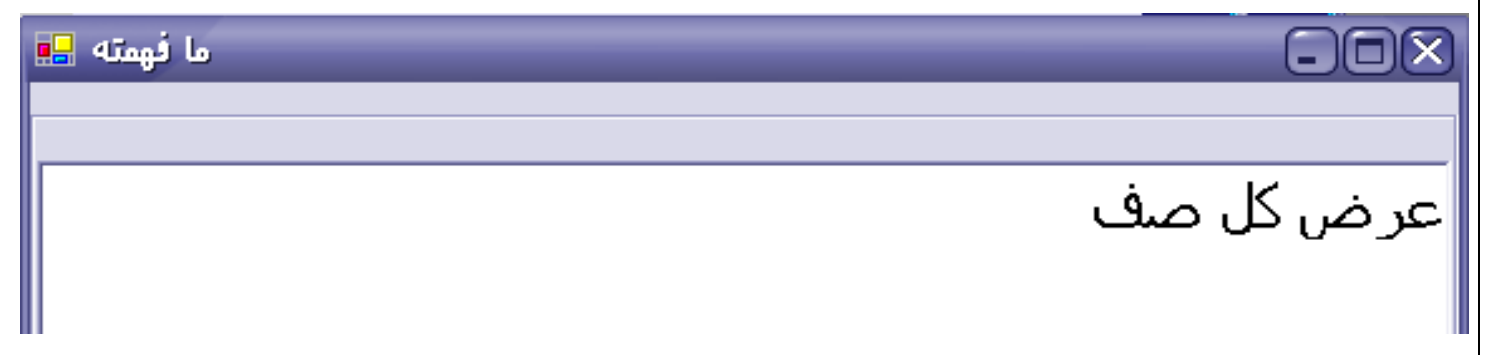


الحالة الثالثة :
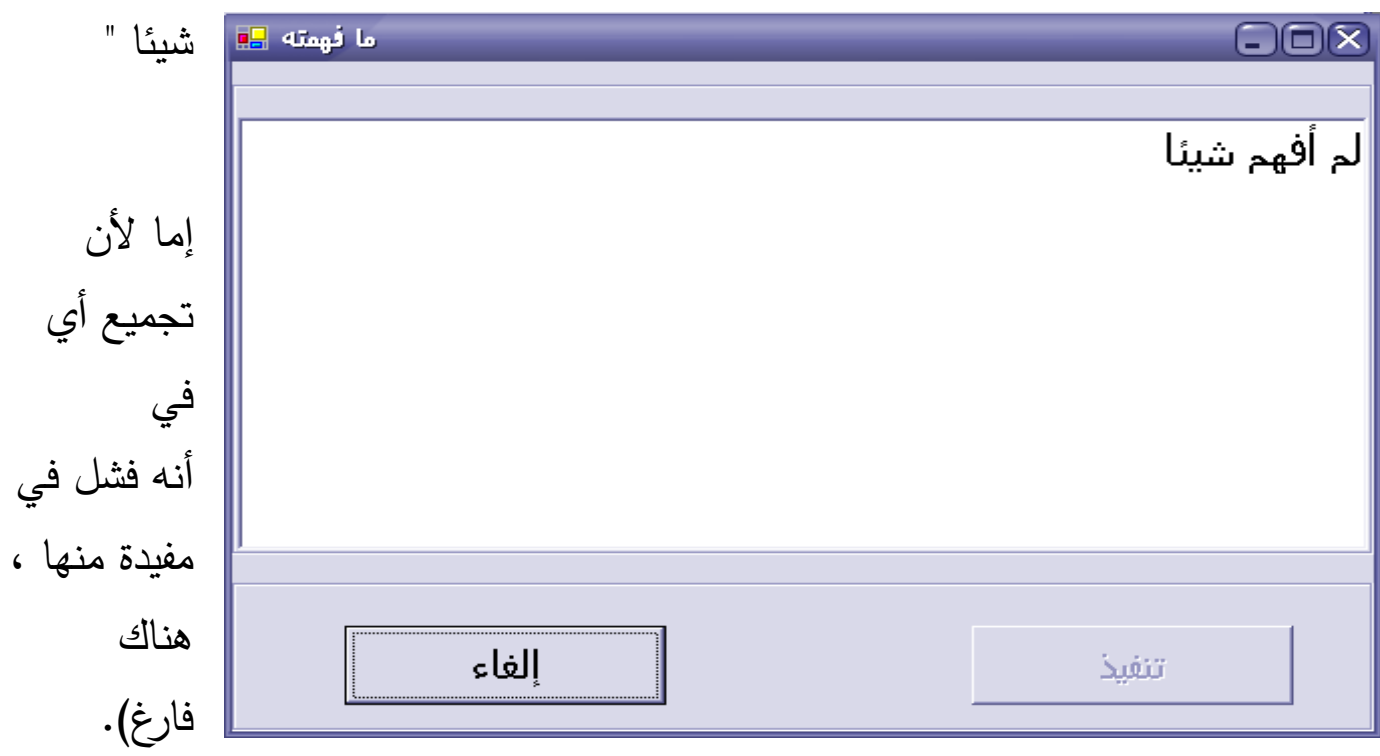

"لم أفهم

الأسباب

النظام لم يستطع

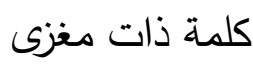
المدخلات ، أي تكوين جملة أو لأنه ليس مدخلات (سطر

4-3 خدمات

أولا : تخزين ناتج الفهح
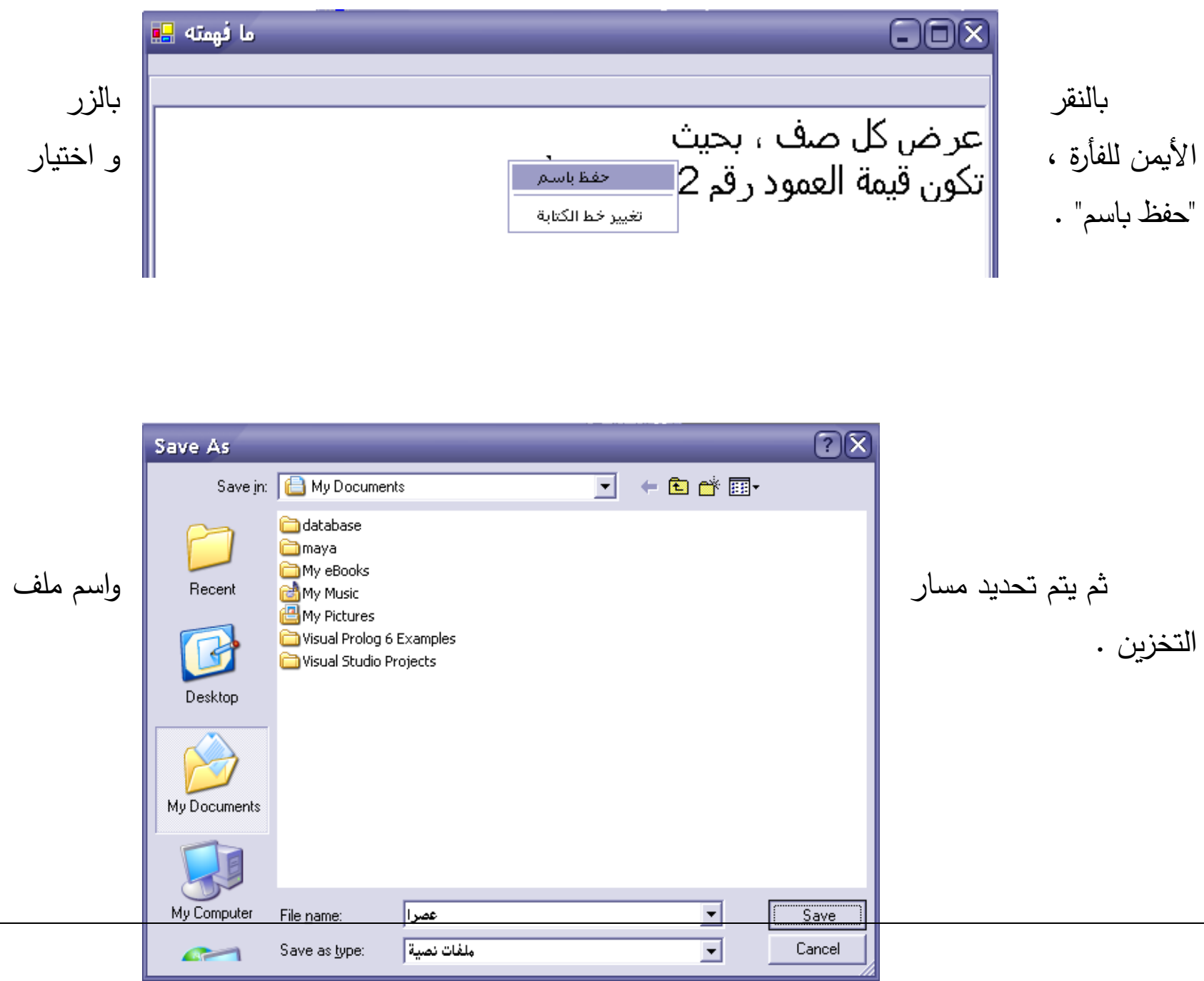
ثم يتم ضغط الزر "Save" .

ثانيا : تهيئة ناتج الفهم
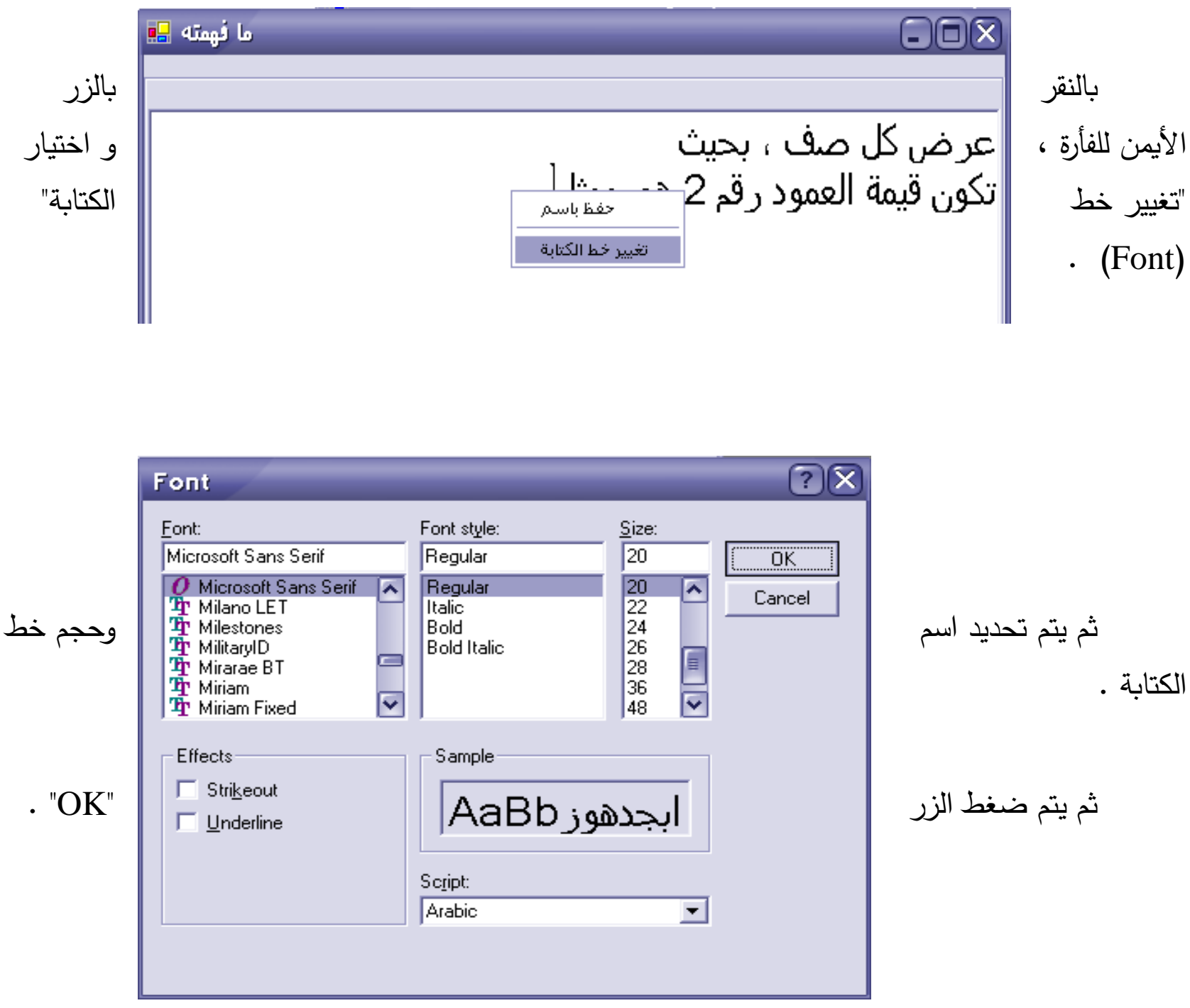

ثالثا : الحافظة (Clipboard)

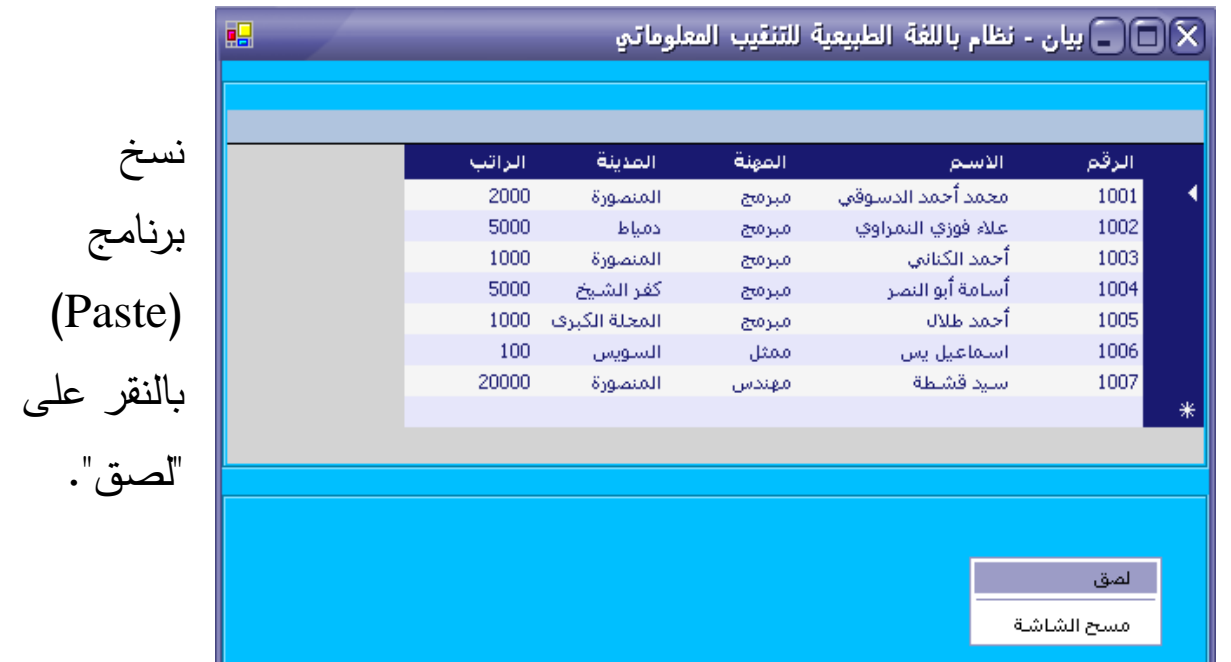

$$
\begin{aligned}
& \text { يمكن القيام بعملية } \\
& \text { لنص من أي لنكن آنصاي } \\
& \text { آخر، ثم عمل لصق } \\
& \text { على الثاشة الرئيسية،وذلك } \\
& \text { الزر الأيمن للفأرة ، و اختيار }
\end{aligned}
$$


وهذا يوفر وقت كتابة جمل الاستعلام 


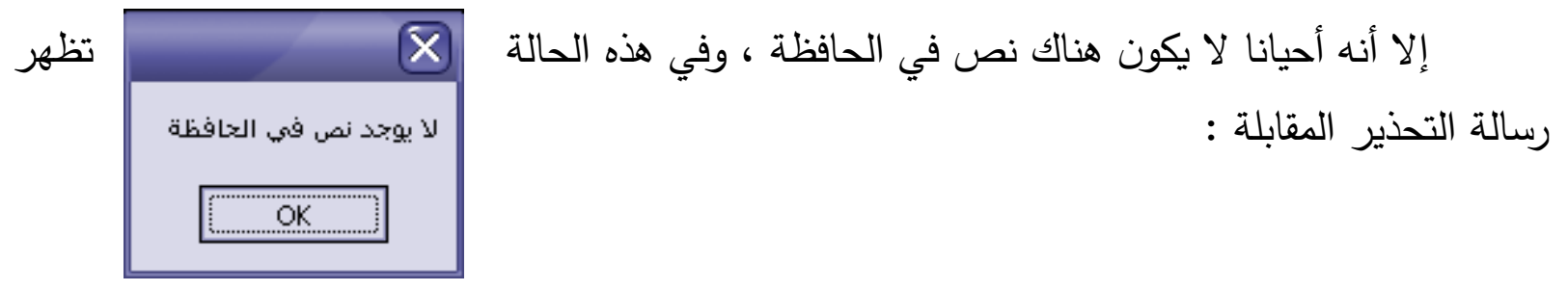

رابعا : مسح الثاشة (مسح منطقة الاستعلام)

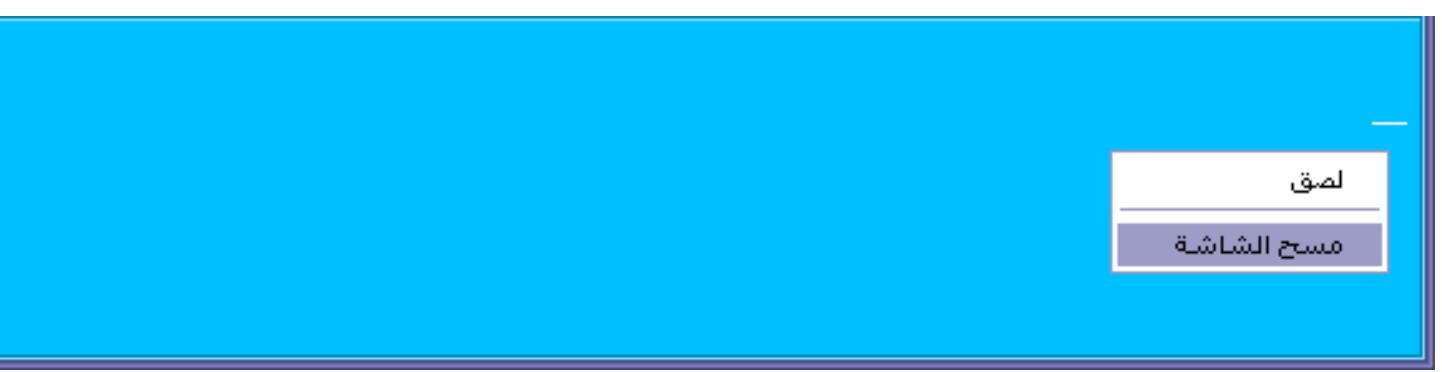

بالنقر بالزر الأيمن للفأرة ، و اختيار " مسح الثاشة" .

خامسا : إدخال صف
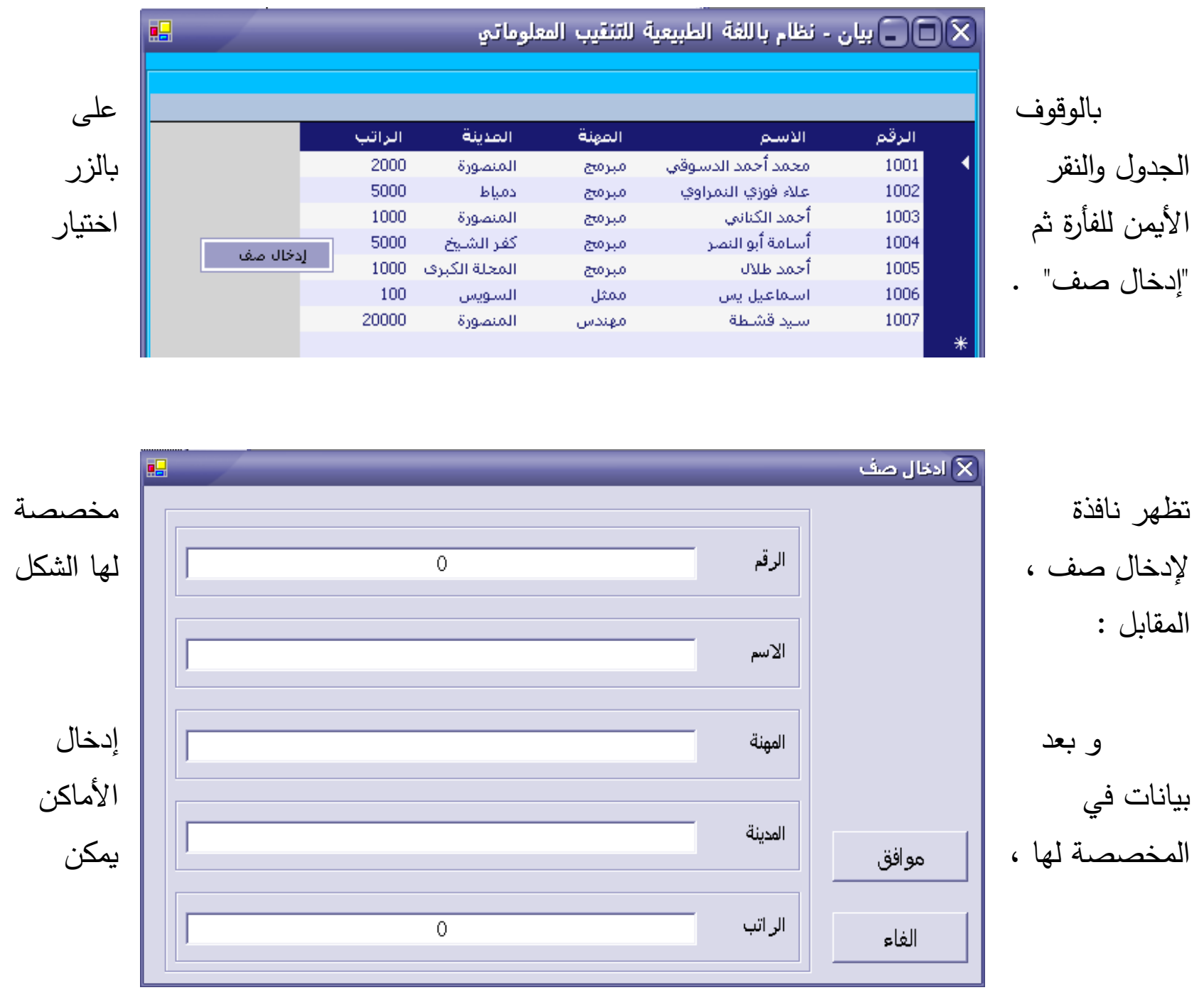
الضغط على زر "موافق" لمتابعة الإدخال أو ضغط زر "إلغاء" للتجاهل .

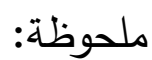

يتوقع النظام أنواع بيانات معينة لتناسب الخلايا ، فعلى سبيل المثال ، عند إدخال القيمة "هـهـهـه

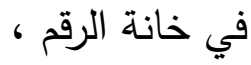
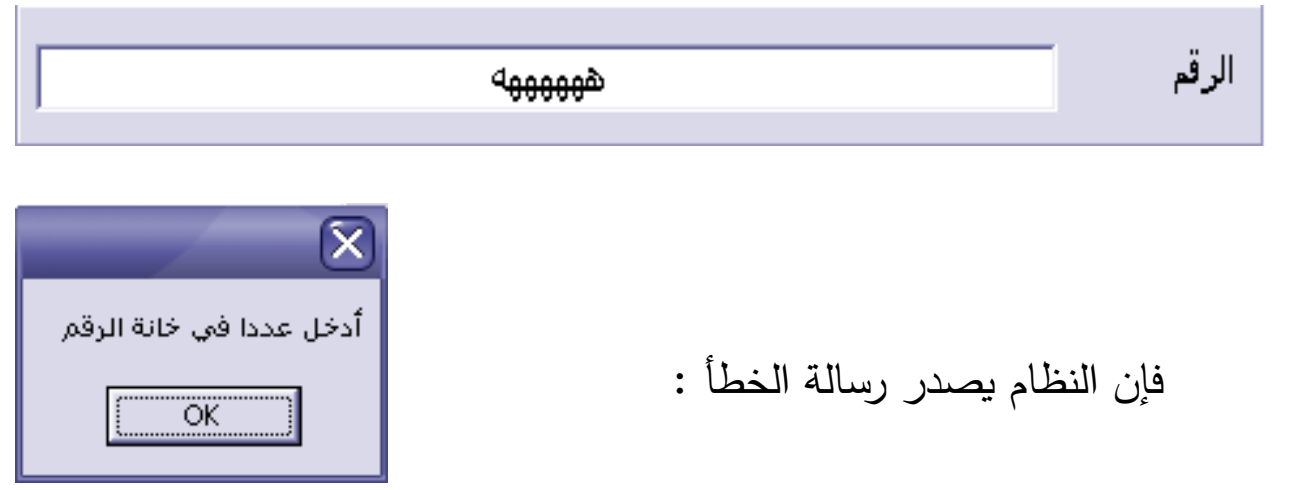

فإن النظام يصدر رسالة الخطأ : (20)

وبالمثل ، عند إدخال القيمة "-45" في خانة الرقم ،

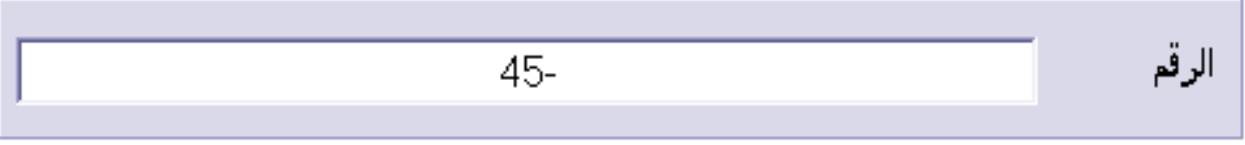

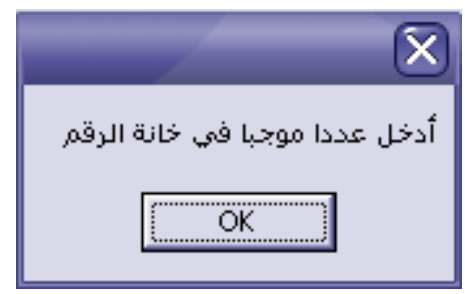

فإن النظام يصدر رسالة الخطأ:

\section{4 التقنيات البرمجية}

\section{1-4 تحليل الكلمات المفتاحية (Keyword Analysis)}

تحليل الكلمات المفتاحية هو من أثهر التقنيات في مجال معالجة اللغات الطبيعية ، حيث يتم

البحث عن كلمات معينة في الجملة، وإعطاء الاستجابة المناسبة لكل كلمة .ويعد تحليل الكلمات المفتاحية مفيدا

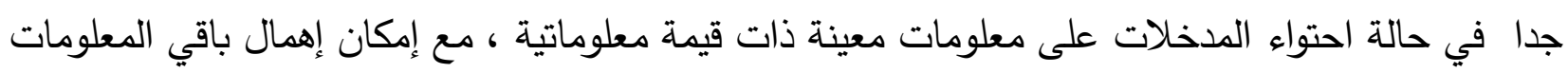

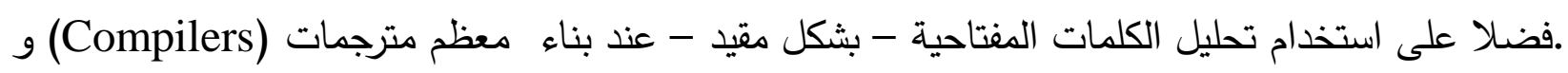
مفسرات (Interpreters) لغات البرمجة الحالية . 
وبمقارنة طريقة تحليل الكلمات المفتاحية بطريقة مضاهاة الأنماط، فإن الأولى تمتاز بالسرعة

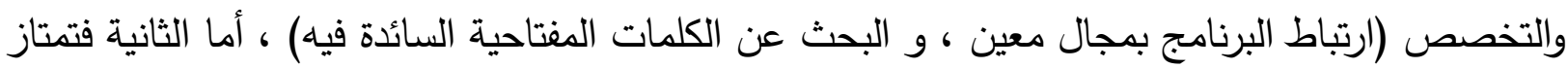
بالشمولية و المساهمة ( إعطاء استجابة تتوقف على كل كلمات المدخل، لا على مجموعة جزئية منه ) .

قبل الشروع في تصميم النظام ، كان هنالك الكثير من التحليل (Analysis) ، تم فيه تحديد الأشكال المختلفة للمدخلات ، و محاولة إيجاد الروابط والعلاقات بين الكلمات ، وتحديد الكلمات المفتاحية .

وهناك ثلاث خطوات رئيسية - بعد تقطيع الدخخلات إلى كلمات- لبناء نظم تحليل الكلمات المفتاحية بوجه عام ، وهذا النظام بوجه خاص، وهي :

\section{1 التبسيط (Simplification)}

وهي خطوة اختيارية ، ويتم فيها توحيد شكل الكلمات ، بمعنى إعطاء الكلمات المتناظرة صورة واحدة ، وهذا يقلل من وقت المقارنة والبحث عن الكلمات المفتاحية.

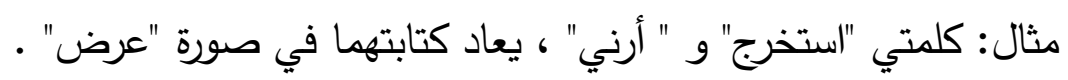

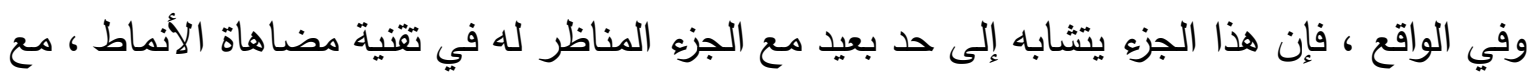

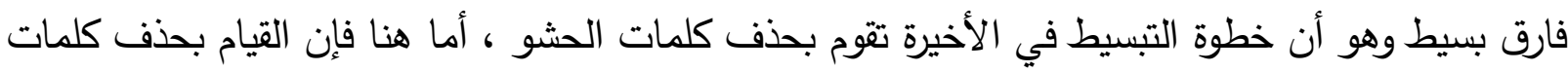

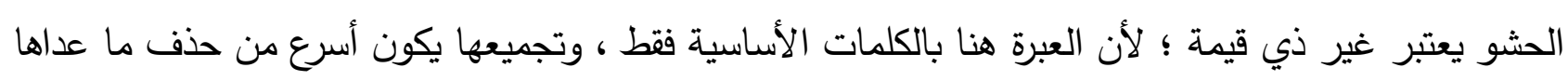

\section{2- تجميع الكلمات المفتاحية (Collecting)}

و الكلمات المفتاحية تختلف باختلاف التطبيق ، وهي في هذا النظام واحدة من اثثين :

$$
\text { عملية (عرض - حذف - تعديل ، ... ) . }
$$

شروط (قيمة خلية ، أو اسم عمود ، أو علاقات على قيمة خلية) .

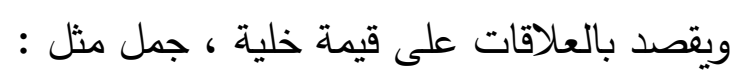

أكبر من ، أصغر من ، أصغر من أو يساوي ،لا تساوي، تساوي، يزيد عن ، ، تقل عن ، ... الخ .

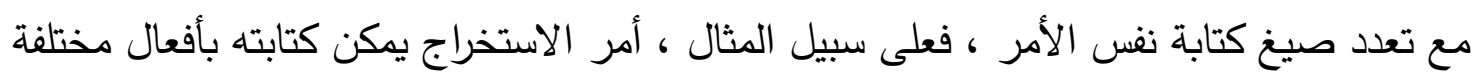

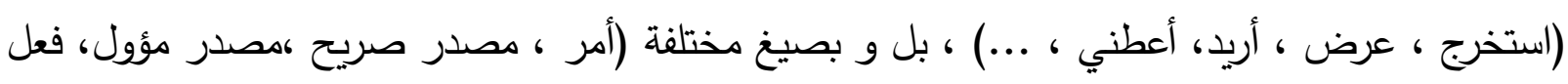

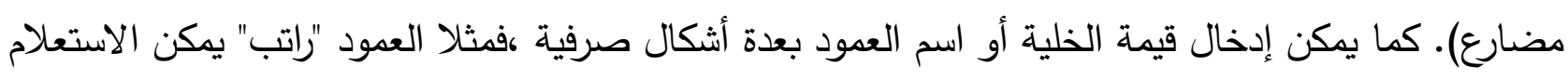

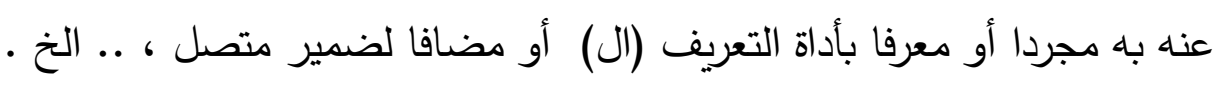


ولهذا، فقد كان على هذا الجزء أن يقوم بعمل تحليل صرفي متقدم ، حتى لا يتجاهل كلمة مفتاحية ، ظنا منه أنها ليست كذلك ثم يتضح أنها مجرد صورة صرفية من كلمة مفتاحية.

\section{(Understanding) الفهم}

ويتم فيها تكوين الاستعلام المراد تتفيذه ، عن طريق ترتيب الكلمات المفتاحية في شكل مقبول ، وبعمل

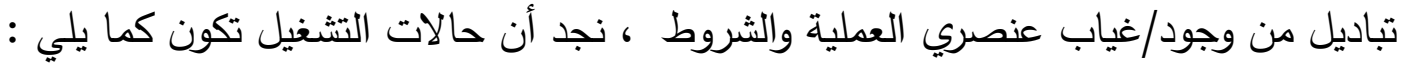

\begin{tabular}{|c|c|c|}
\hline التصرف & شروط & عملية \\
\hline الحالة العادية . & 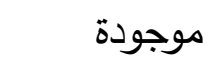 & 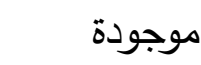 \\
\hline بلا عملية ، لا تتفيذ . & 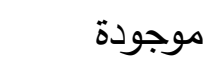 & غير موجودة \\
\hline افتراض العملية على كل الصفوف . & غير موجودة & 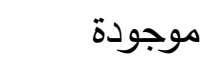 \\
\hline بلا مدخلات تقرببا . & غير موجودة & غير موجودة \\
\hline
\end{tabular}

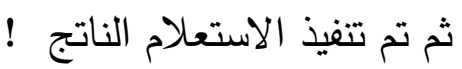

5

\section{1-5 تنظيم الكود}

تم تتظيم الكود في عدة حزم تظهر في الصورة في شكل مجلدات ، تحتوي كل حزمة على ملفات متكاملة في الخصائص الوظيفية .

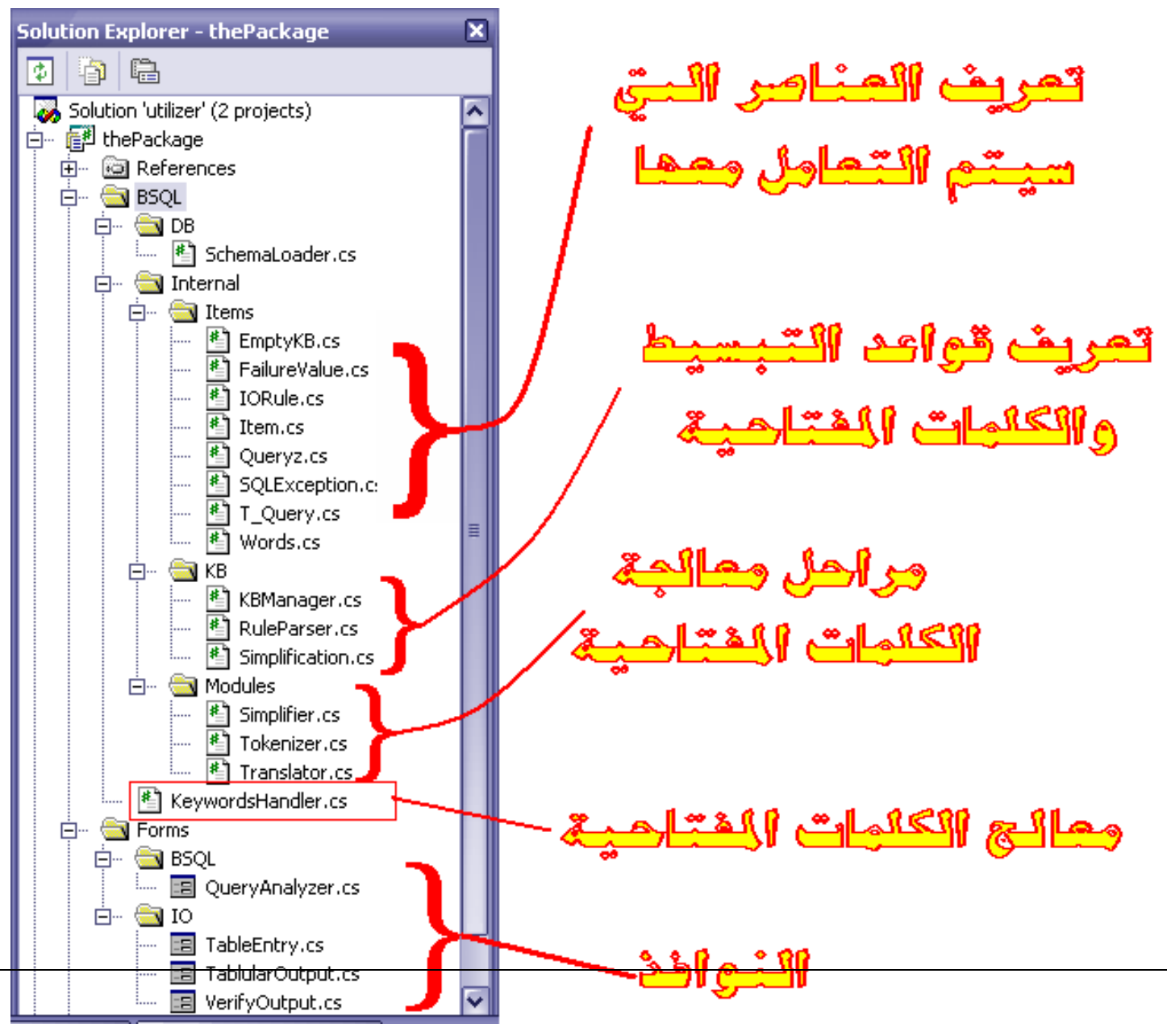




\section{2-5 عرض الكود ( Code Listing}

\section{ملحوظة : - م}

تم عرض الأجزاء الأكثر أهمية فقط ، تاركين الأجزاء الأخرى بدون عرض ، ويمكن الرجوع إليها في الأسطوانة الملحقة . وأهمية هذه الأجزاء تتشأ من أنها تعرض العمليات الأساسية ، كما أنها تقدم فكرة لا بأس بها عن

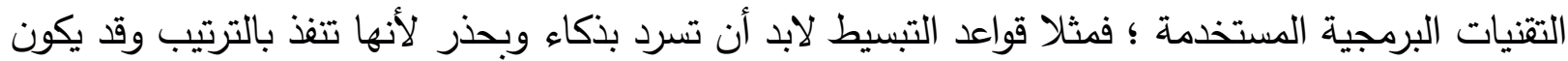
لتنفيذ قاعدة أضرار جانبية على قاعدة أخرى .

\section{أولا : قواعد التبسيط :}

namespace thePackage.BSQL.Internal.KB

\{

public class Simplification

\{

public Arraylist Rules \{get \{return RuleParser.parse_rules(this.TextualRules); \}

string[] TextualRules

$$
\begin{aligned}
& \text { \{get } \text { return new string[] \{ } \\
& \text { "[ }[\text { | \$X] - \$X", } \\
& \text { / / SELECT }
\end{aligned}
$$

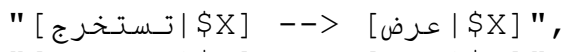

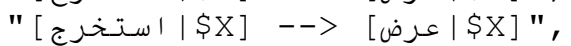

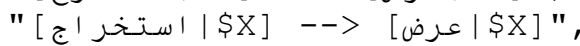

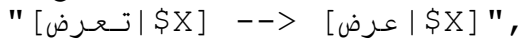

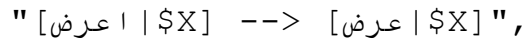

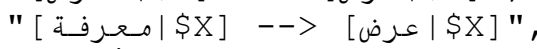

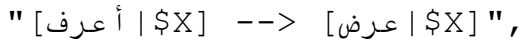

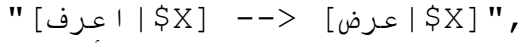

$$
\begin{aligned}
& \text { "] أرى | } \mid \text { | }
\end{aligned}
$$

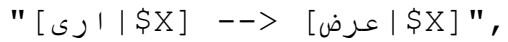

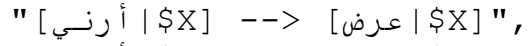

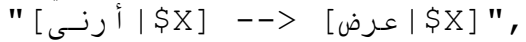

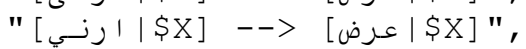

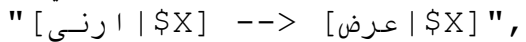

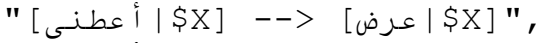

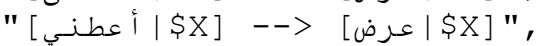

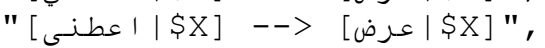

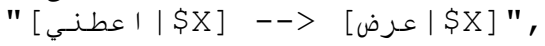

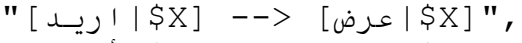

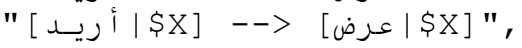

$$
\begin{aligned}
& \text { / /UPDATE }
\end{aligned}
$$

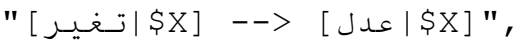

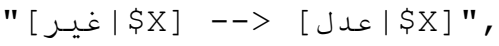

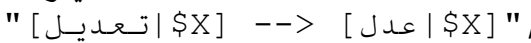

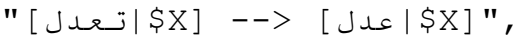

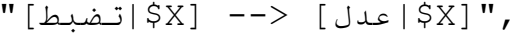

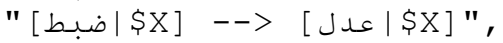

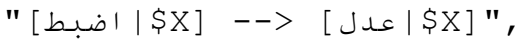

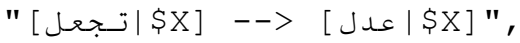

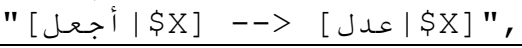




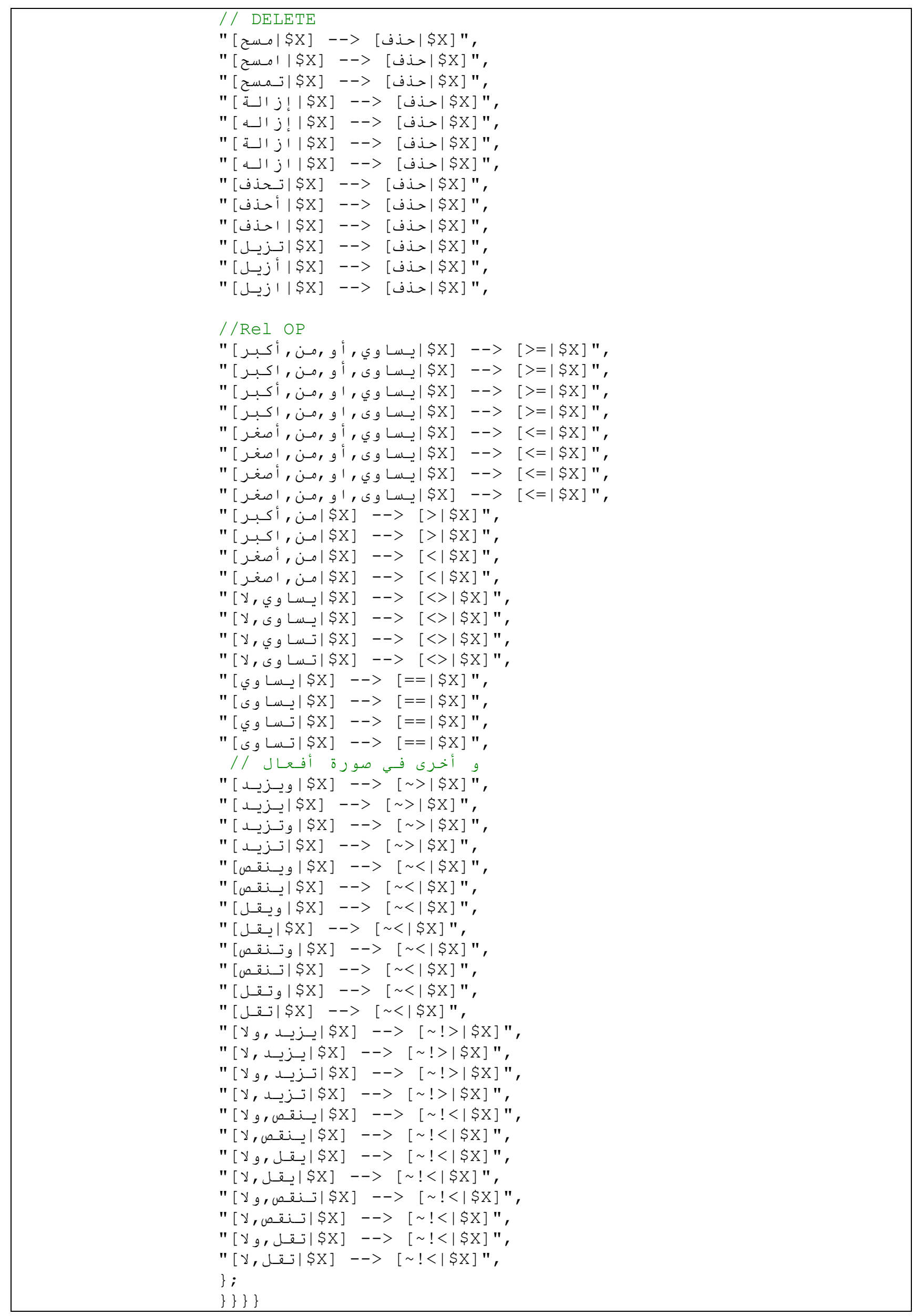




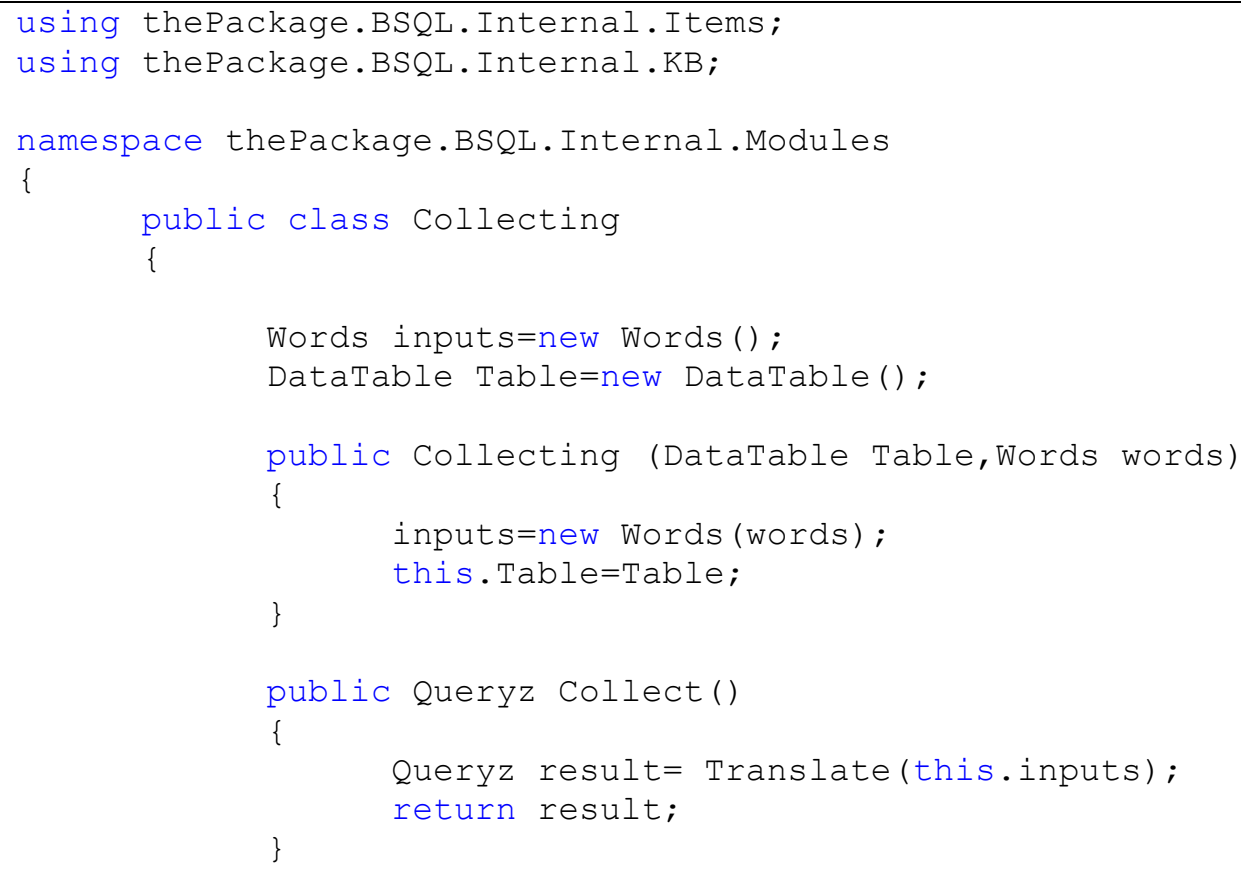




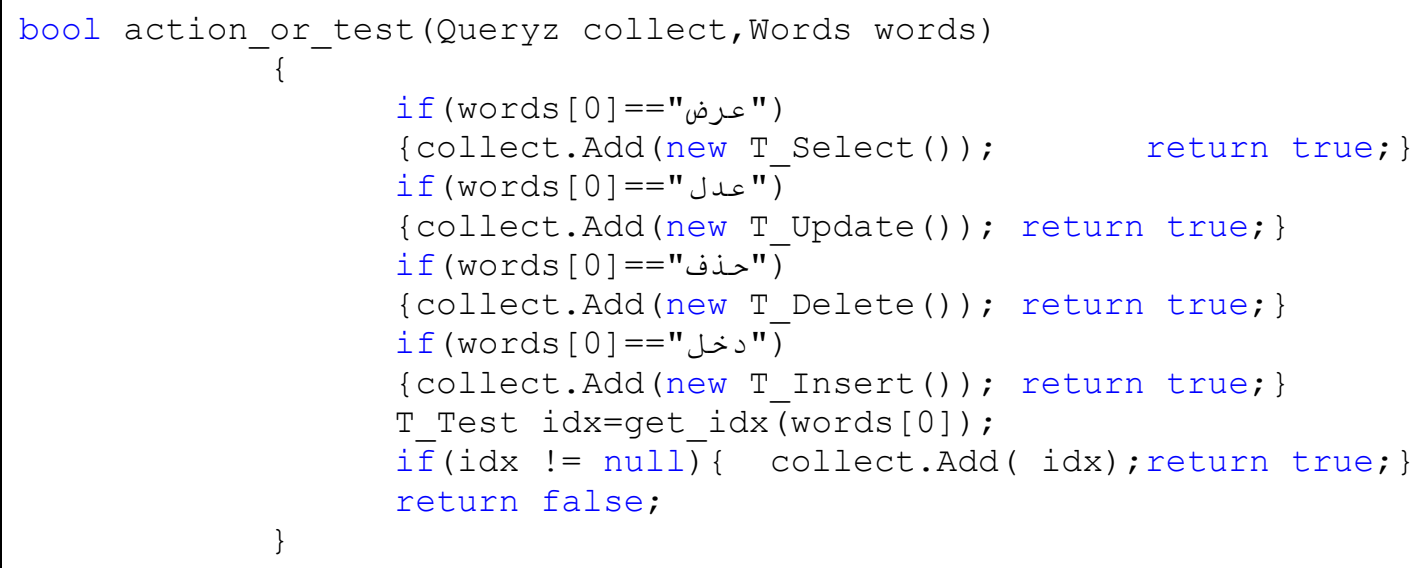

bool comparison(Queryz collect, Words words)

\{

if (words. Items. Length>=3)

\{

if( is_rel_op ( words[0] ) \&\& words[0].StartsWith(" ") )

RelOP op = get_rel_op (words[0]);

$\mathrm{T}$ Argument $\arg 1=$ get argument (words [1]);

$\mathrm{T}$-Argument arg_2= get argument (words [2]);

cōllect.Add (new T_Comparison (op,arg_1,arg_2)); return true;

else if (is_rel_op ( words[1]) \&\& ! words[1].StartsWith(" ") )

Relop op= get rel op (words [1]);

T_Argument arg_1 $=-$ get_argument (words[0]);

T_Argument arg_2= get_argument (words[2]);

cōllect.Add (new T_Comp̄arison (op,arg_1,arg_2));

return true;

\}

\}

return false;

bool is_rel_op (string word)

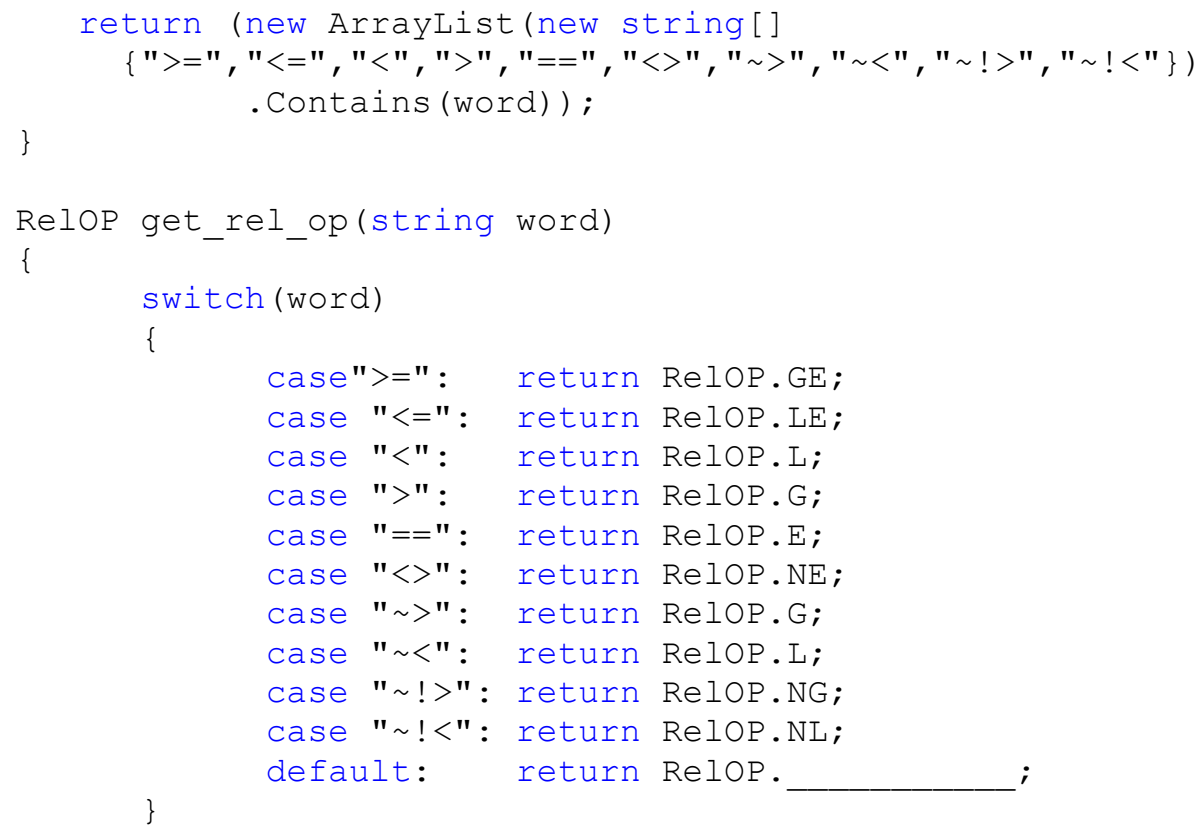


T_Test get_idx(string cellvalue)

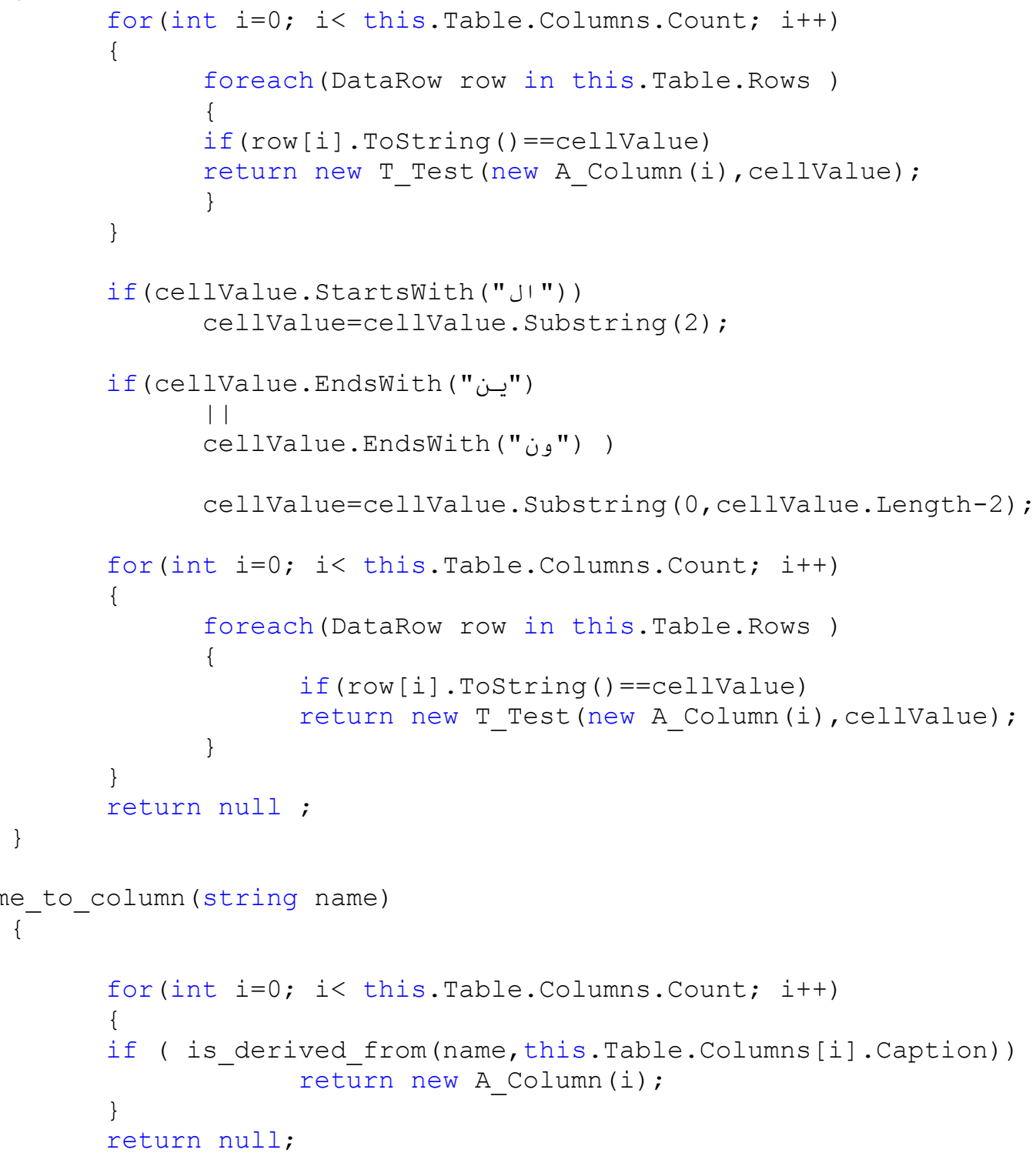


T_Argument get_argument (string word)

A_Column col=name_to_column (word);

if $(\operatorname{col} !=$ null)

return

$\mathrm{COl}$

else return

new A_Value (word);

\}

\section{ثالثا : محلل قواعد التبسيط (RuleParser):}

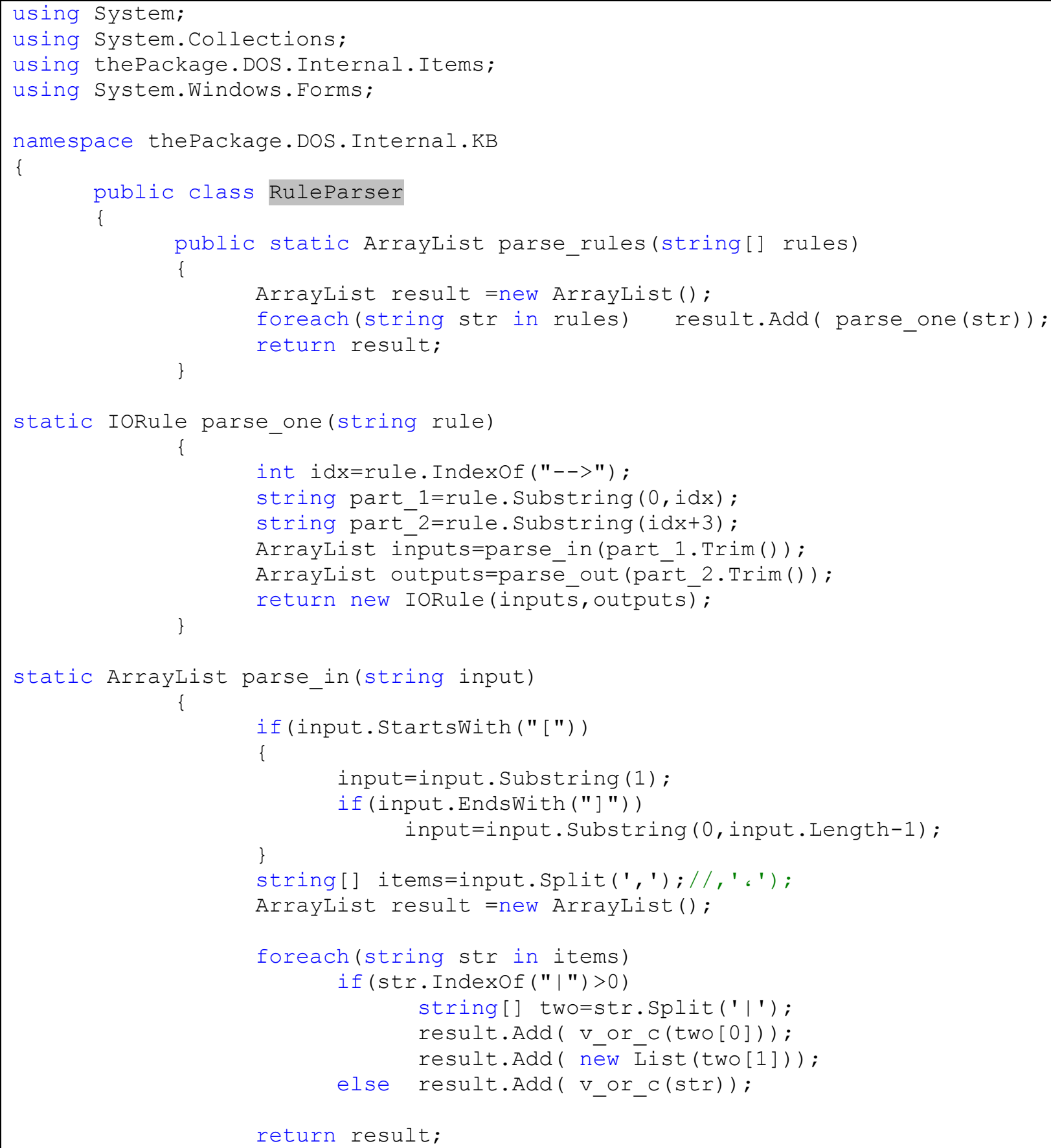




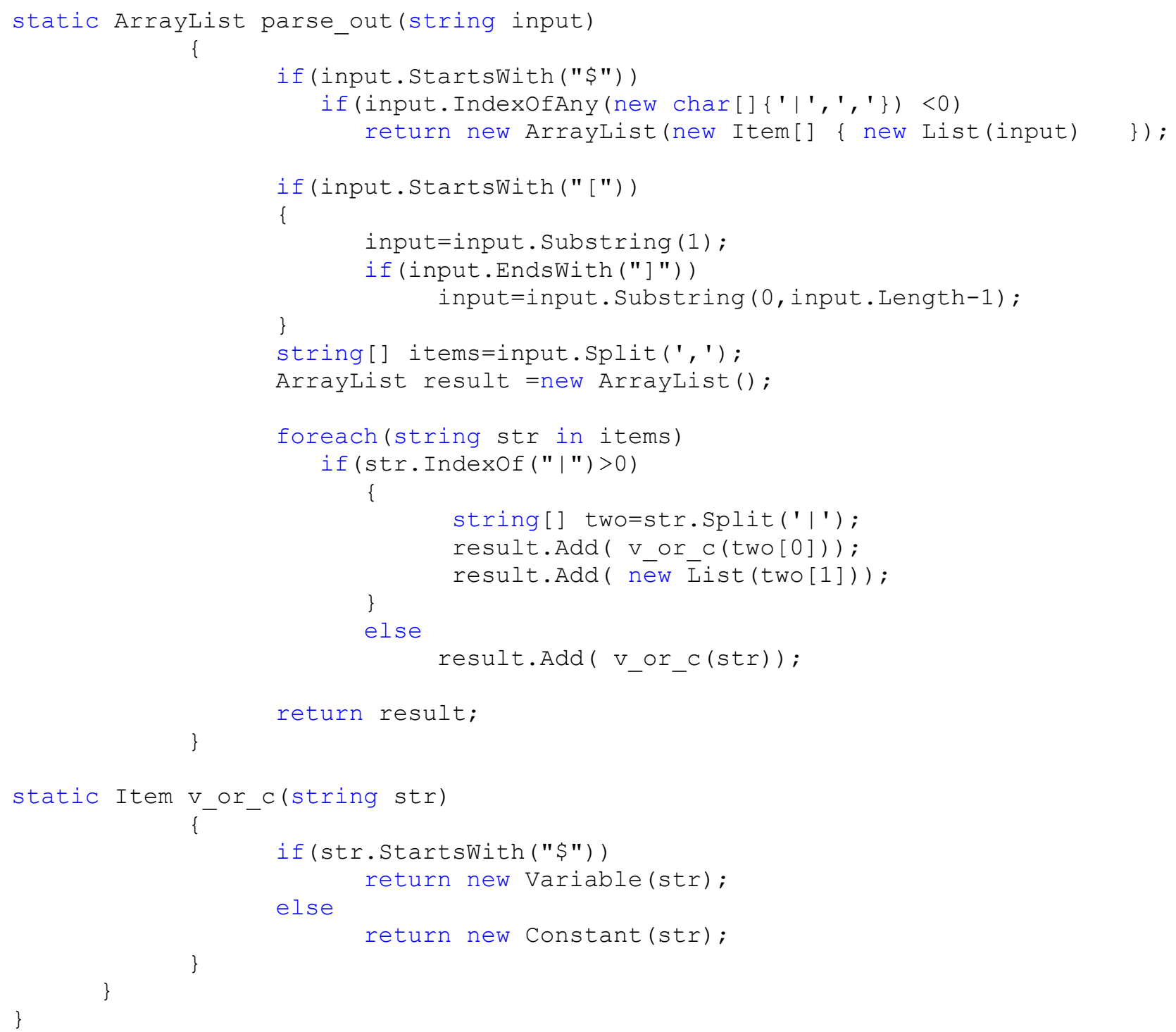

رابعا : المعالج (KeywordsHandler):

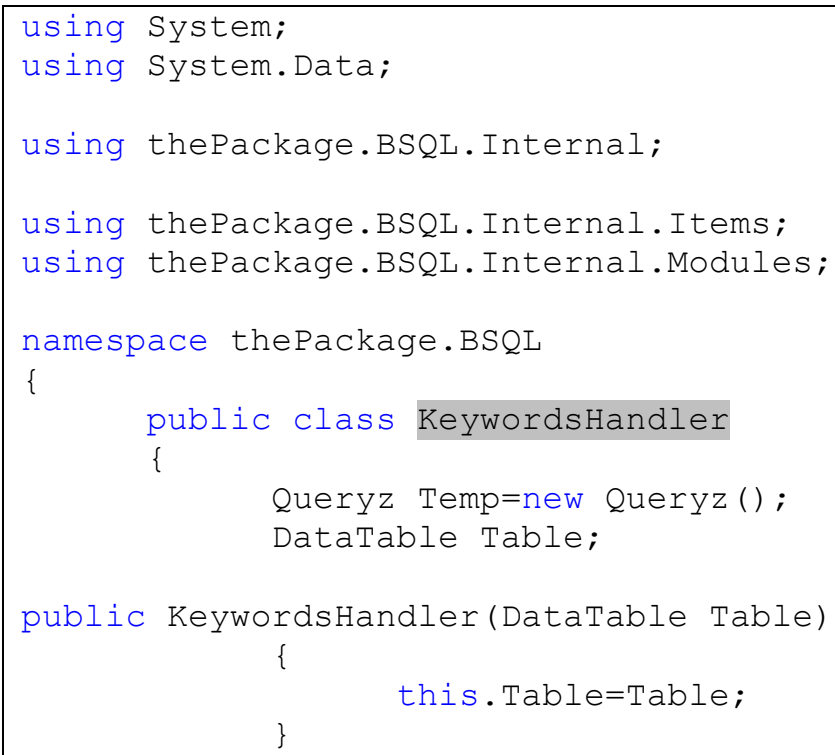


public Queryz Understand(string query)

\{

$$
\text { try }
$$

Queryz result $=$ Collect ( Simplify ( Tokenize (query)) ); \} return result;

catch \{\}

return //new Resultant("تـتـول مـا أفهم لـم") ; new Queryz();

public Words Tokenize(string message)

Tokenizer tokenizer=new Tokenizer(message);

\}

return tokenizer.ToWords ();

public Words Simplify(Words words)

Simplifier simplifier=new Simplifier(words);

\}

return simplifier.Simplify();

public Queryz Collect(Words words)

Collecting collector=new Collecting (this.Table, words);

Queryz result $=$ collector. Collect ();

this.Temp=new Queryz(result);

\}

return result;

\}

خامسا : الثاشة الرئيسية (QueryAnalyzer):

بتصرف، حيث تم إهمال الجزء الخاص بأدوات التحكم ، مع التركيز على كيفية استخدام باقي

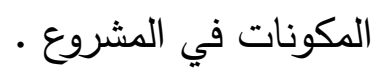

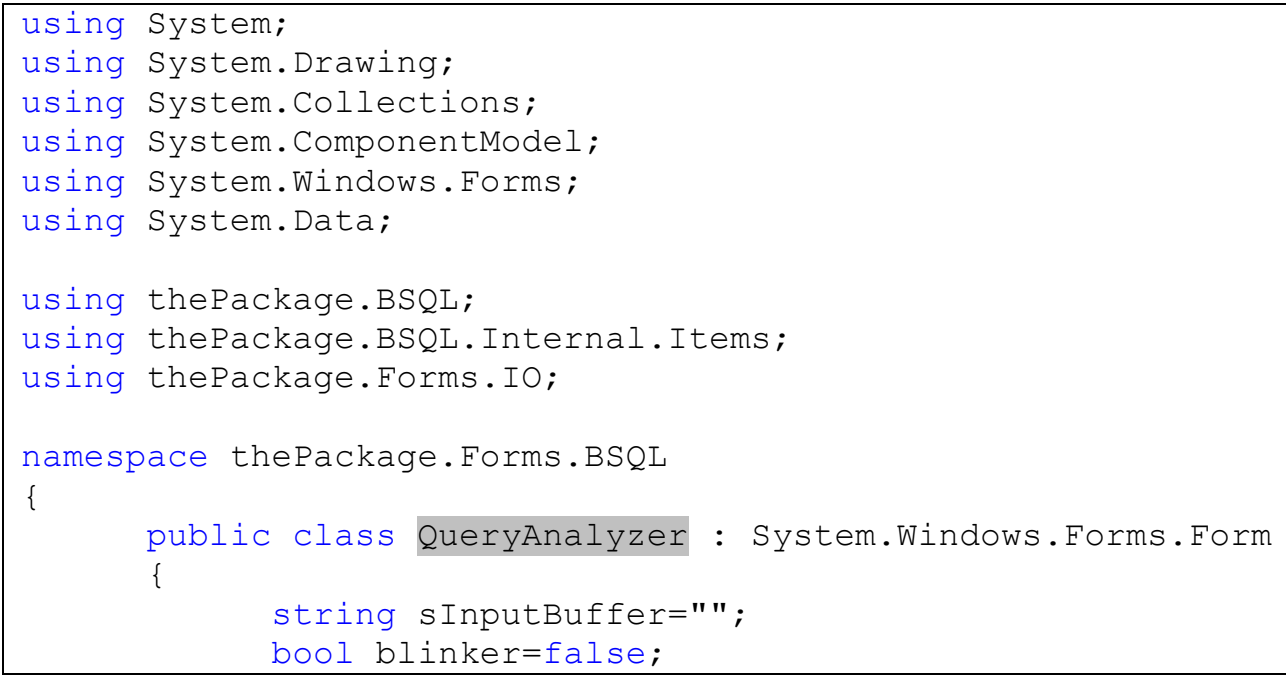




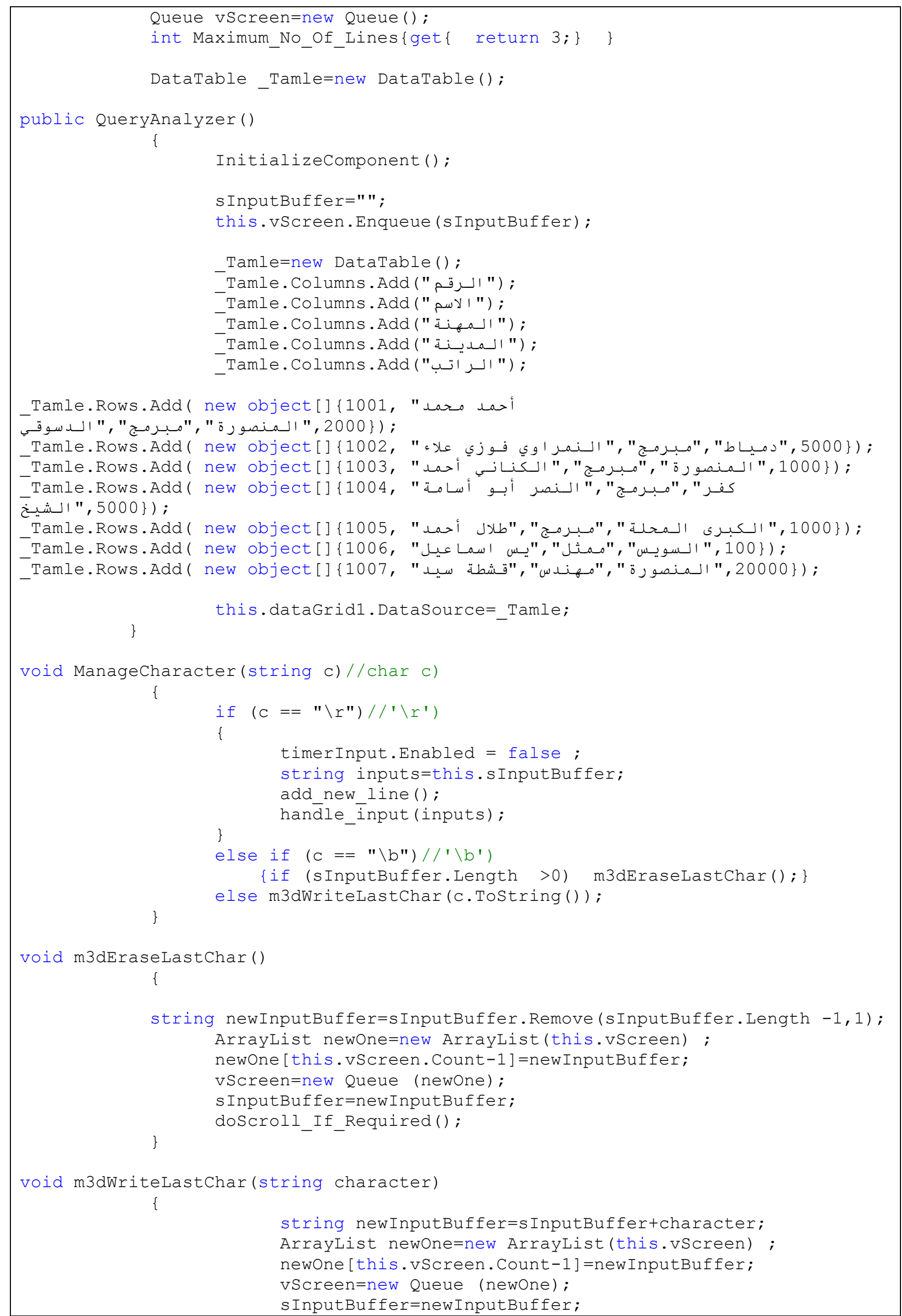


void m3dBlink ()

\{

blinker= ! blinker;

if(blinker) ManageCharacter (" ") ;// ' ');

\}

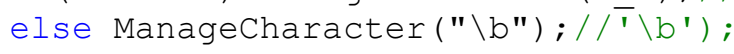

void frmScreen_Closing(object s, System.ComponentModel.CancelEventArgs e) $\{$ this.timerInput.Stop ();

private void Form1_Load(object sender, System.EventArgs e) \{this. ÉmerInput.Start ();

private void timerInput_Tick(object sender, System.EventArgs e) \{

this.textBoxl.Focus(); m3dBlink();

if (this.sInputBuffer. Length >0) doScroll_If Required();

\}

Application.DoEvents ();

void handle_input(string line)

VerifyOutput verify =new VerifyOutput (new

KeywordsHandler(this. Tamle). Understand (line));

if (verify.ShowDialog ()==DialogResult.OK)

\{

try $\quad\{$

Queryz actions=new Queryz();

Queryz test or copmarison=new Queryz();

foreach(T_Query $q$ in verify.my_Queryz.Items)

if( $q$ is T Action) actions. A dd (q); else test_or_copmarison.Add(q);

DataTable res=new DataTable();

res.Columns.Add (" "الصرقـم ") );

res.Columns.Add ("لاسم ") " الاسمة");

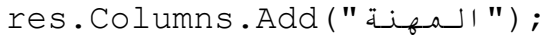

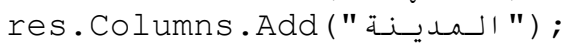

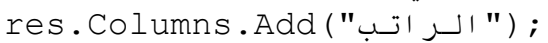

T_Action act $=($ T_Action) actions [0];

if $($ act is T_select)

\{

foreach (DataRow row in this. Tamle.Rows)

if( is_row_match(row, test_or_copmarison)) res.Rows.Add ( row.ItemArray);

if (new TablularOutput (res). ShowDialog ()==DialogResult.OK) \{\}

\}

else if(act is T_Delete)

\{

foreach (DataRow row in this. Tamle.Rows)

if(! is_row match( row, tēst_or_copmarison)) res.Rows.Add ( row.ItemĀrray);

this._Tamle.Rows.Clear ();

foreach (DataRow row in res.Rows)

this._Tamle.Rows.Add (row. ItemArray);

\}

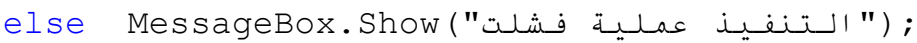




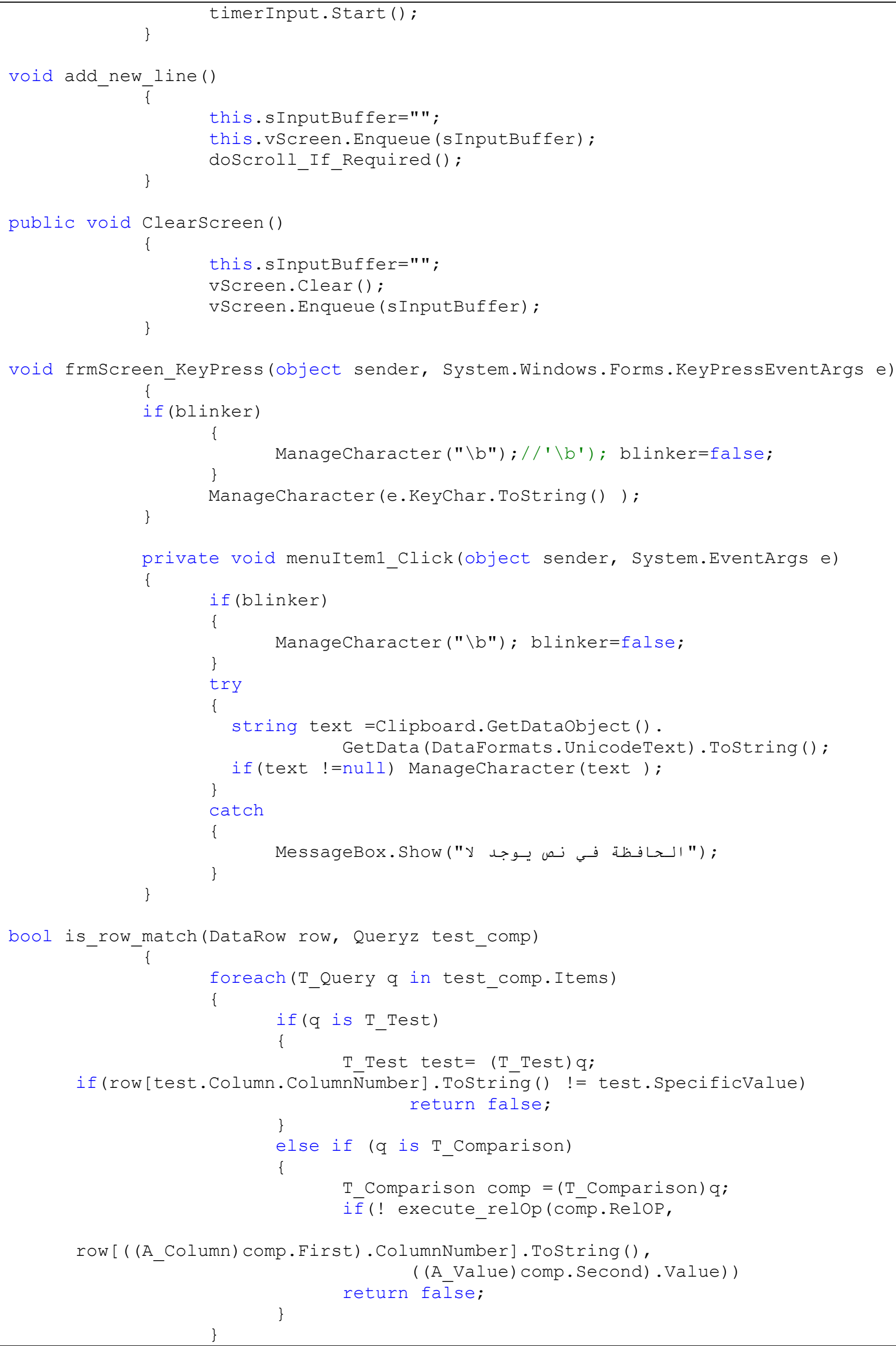


bool execute_relop (Relop op, string arg1, string arg2)

try

int int_1= int.Parse $(\arg 1)$, int_2=int.Parse $(\arg 2)$; switch $(\overline{o p})$

case RelOP.GE: return (int_1 >= int_2);

case RelOP.LE: return (int $1<=$ int 2 );

case RelOP.L: return (int ${ }^{-1}<$ int $\overline{2}$ );

case ReloP.G: return (int_1 > int_2);

case Relop.E: return (int_1 == int_2);

case RelOP.NE: return (int_1 != int_2);

case RelOP.NG: return ! (int_1 $>$ int_2);

case RelOP.NL: return ! (int_1 ${ }_{-}^{-1 n t}{ }_{-}^{2}$ );

\}

catch \{\}

return false;

void menuItem114_Click(object sender, System.EventArgs e)

TableEntry entry=new TableEntry();

if (entry.ShowDialog ( )==DialogResult. OK)

this. Tamle.Rows.Add (entry.Row. ItemArray);

\section{6 ت تطويرات مستقبلية}

o إضافة شروط جديدة ، و بخاصة الثروط المنقدمة ، مثل العمليات الإحصائية (أعلى ، متوسط ، أصغر )،

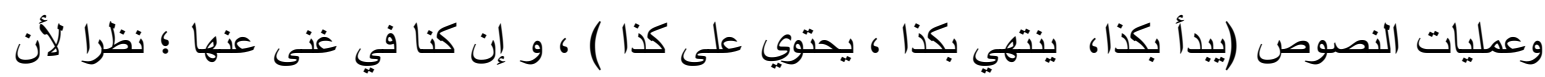
النظام لا يكتفي بالبحث الصارم (Strict) ، بل يقوم بالبحث بعدة صيغ، تمثل أشهر أشكال ورود الكلمات

تصميم النظام ليعمل بالثكل التقليدي للغات قواعد البيانات ، كما في لغة SQL، لتسمح بغرسها (Embedding)

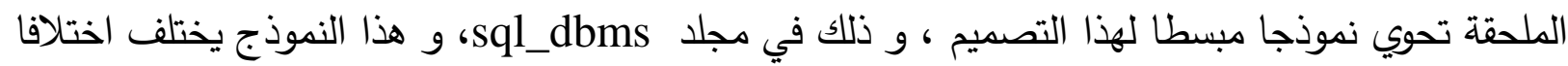
تاما عن النظام الحالي ؛ حيث أن به تعديلات جوهرية تؤهله للعمل كلغة برمجة لقواعد البيانات. 


\section{Bibliography}

\begin{tabular}{|c|c|}
\hline $\begin{array}{l}\text { ADOMAVICIUS } \\
2005\end{array}$ & $\begin{array}{l}\text { ADOMAVICIUS, Gediminas; TUZHILIN, Alexander. Toward } \\
\text { the next generation of recommender systems: A survey of the } \\
\text { state-of-the-art and possible extensions. IEEE Transactions on } \\
\text { Knowledge \& Data Engineering, 2005, 6: 734-749. }\end{array}$ \\
\hline Allthingslinguistic & $\begin{array}{l}\text { https://allthingslinguistic.com/post/52411342274/how-many- } \\
\text { meanings-can-you-get-for-the-sentence-i }\end{array}$ \\
\hline ARAKI2017 & $\begin{array}{l}\text { ARAKI, Naoki. Chomsky's I-language and E-language. } \\
\text { 広島工業大学紀要. 研究編, 2017, 51: 17-24. }\end{array}$ \\
\hline AUSTIN1975 & $\begin{array}{l}\text { AUSTIN, John Langshaw. How to do things with words. Oxford } \\
\text { university press, } 1975 .\end{array}$ \\
\hline BERLO1977 & $\begin{array}{l}\text { BERLO, David K. Communication as process: Review and } \\
\text { commentary. Annals of the International Communication } \\
\text { Association, 1977, 1.1:11-27. }\end{array}$ \\
\hline Blitzstei & $\begin{array}{l}\text { Blitzstein et al, Probability Cheat sheet } \\
\text { http://www.wzchen.com/probability-cheatsheet } 2015\end{array}$ \\
\hline CELTA2007 & $\begin{array}{l}\text { THORNBURY, Scott; WATKINS, Peter. The CELTA course: } \\
\text { Trainee book. Ernst Klett Sprachen, } 2007 \text {. }\end{array}$ \\
\hline CHOMSKY1957 & CHOMSKY, Noam. The structure of language. 1957. \\
\hline CHOMSKY1986 & $\begin{array}{l}\text { CHOMSKY, Noam. Knowledge of language: Its nature, origin, } \\
\text { and use. Greenwood Publishing Group, } 1986 .\end{array}$ \\
\hline COLLINS1999 & $\begin{array}{l}\text { COLLINS, Michael, et al. A statistical parser for Czech. In: } \\
\text { Proceedings of the } 37 \text { th annual meeting of the Association for } \\
\text { Computational Linguistics on Computational Linguistics. } \\
\text { Association for Computational Linguistics, 1999.p. 505-512. }\end{array}$ \\
\hline $\begin{array}{l}\text { COVINGTON199 } \\
4\end{array}$ & $\begin{array}{l}\text { COVINGTON, Michael A. Natural language processing for } \\
\text { Prolog programmers. Englewood Cliffs (NJ): Prentice hall, } \\
1994 \text {. }\end{array}$ \\
\hline COVER2012 & $\begin{array}{l}\text { COVER, Thomas M.; THOMAS, Joy A. Elements of } \\
\text { information theory. John Wiley \& Sons, } 2012 .\end{array}$ \\
\hline $\begin{array}{l}\text { DESAUSSURE19 } \\
89\end{array}$ & $\begin{array}{l}\text { DE SAUSSURE, Ferdinand. Cours de linguistique générale: } \\
\text { Édition critique. Otto Harrassowitz Verlag, } 1989 .\end{array}$ \\
\hline DICT & http://www.cse.unsw.edu.au/ billw/nlpdict.html \\
\hline Dummies & $\begin{array}{l}\text { https://www.dummies.com/education/math/probability/probabili } \\
\text { ty-for-dummies-cheat-sheet/ }\end{array}$ \\
\hline $\begin{array}{l}\text { EL- } \\
\text { DOSUKY2012 }\end{array}$ & $\begin{array}{l}\text { EL-DOSUKY, M. A., et al. Food recommendation using } \\
\text { ontology and heuristics. In: International conference on } \\
\text { advanced machine learning technologies and applications. } \\
\text { Springer, Berlin, Heidelberg, 2012.p. 423-429. }\end{array}$ \\
\hline EVANS2011 & EVANS, Nicholas. Dying words: Endangered languages and \\
\hline
\end{tabular}




\begin{tabular}{|c|c|}
\hline & what they have to tell us. John Wiley \& Sons, 2011. \\
\hline Gardiner1927 & $\begin{array}{l}\text { Gardiner A.H., Egyptian Grammar: Being an Introduction to the } \\
\text { Study of Hieroglyphs. 3rd Ed., pub. Griffith Institute, Oxford, } \\
1957 \text { (1st edition 1927), pp. 438-548 } \\
\text { And } \\
\text { Egypt of the Pharaohs By Sir Alan Gardiner(In Arabic) }\end{array}$ \\
\hline GloVe & $\begin{array}{c}\text { Global Vectors for Word Representation (GloVe, } \\
\text { https://nlp.stanford.edu/projects/glove/ }\end{array}$ \\
\hline $\begin{array}{l}\text { HUCKVALE200 } \\
8\end{array}$ & $\begin{array}{l}\text { HUCKVALE, Mark. Make your own vowel resonators. } 2008 . \\
\text { https://www.phon.ucl.ac.uk/home/mark/vowels/ }\end{array}$ \\
\hline IGNATOW2016 & $\begin{array}{l}\text { IGNATOW, Gabe; MIHALCEA, Rada. Text mining: A } \\
\text { guidebook for the social sciences. Sage Publications, } 2016 .\end{array}$ \\
\hline IPA1999 & $\begin{array}{l}\text { DECKER, Donald M., et al. Handbook of the International } \\
\text { Phonetic Association: A guide to the use of the International } \\
\text { Phonetic Alphabet. Cambridge University Press, } 1999 .\end{array}$ \\
\hline ITU 2009 & $\begin{array}{l}\text { International Telecommunication Union, Morse Code } \\
\text { www.itu.int } 2009\end{array}$ \\
\hline JAK & $\begin{array}{l}\text { JAKOBSON, Roman (ed.). Structure of language and its } \\
\text { mathematical aspects. American Mathematical Soc., } 1961 .\end{array}$ \\
\hline KY2014 & $\begin{array}{l}\text { JURAFSKY, Dan; MARTIN, James H. Speech and language } \\
\text { processing. London: Pearson, } 2014 .\end{array}$ \\
\hline $\mathrm{KOE}$ & $\begin{array}{l}\text { KOEHN, Philipp. Statistical significance tests for machine } \\
\text { translation evaluation. In: Proceedings of the } 2004 \text { conference } \\
\text { on empirical methods in natural language processing. } 2004 \text {. }\end{array}$ \\
\hline $\begin{array}{l}\text { LANGACKER19 } \\
87\end{array}$ & $\begin{array}{l}\text { LANGACKER, Ronald W. Foundations of cognitive grammar: } \\
\text { Theoretical prerequisites. Stanford university press, } 1987 .\end{array}$ \\
\hline LEE2008 & $\begin{array}{l}\text { LEE, Dongjoo; JEONG, Ok-Ran; LEE, Sang-goo. Opinion } \\
\text { mining of customer feedback data on the web. In: Proceedings } \\
\text { of the 2nd international conference on Ubiquitous information } \\
\text { management and communication. ACM, 2008. p. 230-235. }\end{array}$ \\
\hline $\begin{array}{l}\text { Linguisticsinactio } \\
\mathrm{n}\end{array}$ & $\begin{array}{l}\text { https://sites.google.com/site/linguisticsinaction/linguistics/histor } \\
\text { y-of-linguistics-1/study-of-sociolinguistics }\end{array}$ \\
\hline MANNING1999 & $\begin{array}{l}\text { MANNING, Christopher D.; MANNING, Christopher D.; } \\
\text { SCHÜTZE, Hinrich. Foundations of statistical natural language } \\
\text { processing. MIT press, } 1999 \text {. }\end{array}$ \\
\hline Markov & http://homepages.rpi.edu/ sharkt/MarkovChains.html \\
\hline Me.me & $\begin{array}{l}\text { https://me.me/i/hello-are-you-there-yes-who-are-you-im-watt- } \\
21690913\end{array}$ \\
\hline Mediawise & $\begin{array}{l}\text { https://mediawise.net.au/blog/punctuation-difference-between- } \\
\text { life-and-death-grandma }\end{array}$ \\
\hline$N L T K$ & $\begin{array}{l}\text { BIRD, Steven; KLEIN, Ewan; LOPER, Edward. Natural } \\
\text { language processing with Python: analyzing text with the natural } \\
\text { language toolkit. " O'Reilly Media, Inc.", } 2009 . \\
\text { https://www.nltk.org/book/ }\end{array}$ \\
\hline
\end{tabular}




\begin{tabular}{|c|c|}
\hline Ontotext2017 & https://ontotext.com/top-5-semantic-technology-trends-2017/ \\
\hline Openclips & https://openclipart.org \\
\hline PEIRCE2012 & $\begin{array}{l}\text { PEIRCE, Charles S. Philosophical writings of Peirce. Courier } \\
\text { Corporation, } 2012 .\end{array}$ \\
\hline PrintDriver & http://www.print-driver.com/stories/huffman-coding-jpeg \\
\hline SKINNER1957 & $\begin{array}{l}\text { SKINNER, B. F. Century psychology series. Verbal behavior. } \\
\text { East Norwalk, CT, US: Appleton-Century-Crofts. } 1957 . \\
\text { And } \\
\text { FERSTER, Charles B.; SKINNER, Burrhus Frederic. Schedules } \\
\text { of reinforcement. 1957. }\end{array}$ \\
\hline SMITH1977 & $\begin{array}{l}\text { SMITH, Robert W. Sir James Hopwood Jeans, 1877-1946. } \\
\text { Journal of the British Astronomical Association, 1977, 88: 8-17. }\end{array}$ \\
\hline Steemit & $\begin{array}{l}\text { https://steemit.com/bayes/@ sulev/bayes-theorem-how-to-think- } \\
\text { better }\end{array}$ \\
\hline $\begin{array}{l}\text { STERNBERG201 } \\
6 \\
\end{array}$ & $\begin{array}{l}\text { STERNBERG, Robert J.; STERNBERG, Karin. Cognitive } \\
\text { psychology. Nelson Education, } 2016 .\end{array}$ \\
\hline THEDE1999 & $\begin{array}{l}\text { THEDE, Scott M.; HARPER, Mary P. A second-order hidden } \\
\text { Markov model for part-of-speech tagging. In: Proceedings of the } \\
\text { 37th annual meeting of the Association for Computational } \\
\text { Linguistics on Computational Linguistics. Association for } \\
\text { Computational Linguistics, 1999.p. 175-182. }\end{array}$ \\
\hline TITZE1998 & $\begin{array}{l}\text { TITZE, Ingo R.; MARTIN, Daniel W. Principles of voice } \\
\text { production. } 1998 .\end{array}$ \\
\hline Tofugu & https://www.tofugu.com/japanese/hiragana-chart/ \\
\hline Tough & https://www.fluentin3months.com/tough/ \\
\hline TRAGER2009 & $\begin{array}{l}\text { TRAGER, George Leonard; SMITH, Henry Lee. An outline of } \\
\text { English structure. Рипол Классик, } 2009 .\end{array}$ \\
\hline VAUQUOIS1968 & $\begin{array}{l}\text { VAUQUOIS, Bernard. Structures profondes et traduction } \\
\text { automatique. Le système du CETA. Revue Roumaine de } \\
\text { linguistique, } 1968 . \\
\text { And } \\
\text { VAUQUOIS, Bernard. A survey of formal grammars and } \\
\text { algorithms for recognition and transformation in mechanical } \\
\text { translation. In: Ifip congress (2). 1968. p. 1114-1122. }\end{array}$ \\
\hline WALPOLE2014 & $\begin{array}{l}\text { WALPOLE, Ronald E., et al. Probability and statistics for } \\
\text { engineers and scientists. London: Pearson, } 2014 .\end{array}$ \\
\hline Wittgenstein & http://plato.stanford.edu/entries/wittgenstein \\
\hline WRIGHT1954 & $\begin{array}{l}\text { WRIGHT, Sylvia. The Death of Lady Mondegreen. Harper's } \\
\text { Magazine, 1954, 209.1254: 48-51. }\end{array}$ \\
\hline
\end{tabular}

